\title{
On the expressive power of Query languages
}

\section{Report}

Author(s):

Wüthrich, Beat; Schäuble, Peter

Publication date:

1992

Permanent link:

https://doi.org/10.3929/ethz-a-000628399

\section{Rights / license:}

In Copyright - Non-Commercial Use Permitted

\section{Originally published in:}

ETH, Eidgenössische Technische Hochschule Zürich, Departement Informatik, Institut für Informationssysteme 173 


\section{EIH}

Eidgenössische

Technische Hochschule

Zürich
Departement Informatik Institut für Informationssysteme
Peter Schäuble Beat Wüthrich
On the Expressive Power of Query Languages

February 1992 
Authors' addresses:

Peter Schäuble, Institut für Informationssysteme ETH Zentrum, $\mathrm{CH}-8092$ Zurich, Switzerland e-mail: schauble@inf.ethz.ch

Beat Wüthrich, ECRC GmbH

Arabellastrasse 17, D-W-8000 München 81, Germany e-mail: Beat.Wuetrich@ecrc.de

(c) 1992 Departement Informatik, ETH Zürich 


\begin{abstract}
Two main topics are addressed. First, an algebraic approach is presented to define a general notion of expressive power. Heterogeneous algebras represent information systems and morphisms represent the correspondences between the instances of databases, the correspondences between answers; and the correspondences between queries. An important feature of this new notion of expressive power is that query languages of different types can be compared with respect to their expressive power. In the case of relational query languages, the new notion of expressive power is shown to be equivalent to the notion used by Chandra and Harel. In the case of non-relational query languages, the versatility of the new notion of expressive power is demonstrated by comparing a Boolean query language with a weighted query language consisting of vague queries. We also demonstrate that the new notion of expressive power is appropriate to compare the fixpoint query languages with an object-oriented query language called FQL*. The expressive power of the Functional Query Language FQL* is the second main topic of this paper. The specifications of FQL* functions can be recursive or even mutually recursive. FQL* has a fixpoint semantics based on a complete lattice consisting of bag functions. The query language $\mathrm{FQL}^{*}$ is shown to be more expressive than the fixpoint query languages. This result implies that FQL* is also more expressive than Datalog with stratified negation. Examples of recursive FQL* functions are given that determine the ancestors of persons and the bill of materials.
\end{abstract}




\section{Introduction}

The expressive power is one of the important features of a query language. Whether a specific need for information can be expressed in a query language or not depends on its expressive power. A thorough analysis of the expressive power of relational query languages has been presented by Chandra and Harel in [9], [10] (see also [11] for an overview and new results). They consider queries as partial functions mapping relational databases to relations. A query language is characterized by its corresponding set of queries. Considering a query language as a set of partial functions, a query language $Q L^{\prime}$ is as expressive as a query language $Q L$ iff $Q L^{\prime}$ is a superset of $Q L$. Using this notion by Chandra and Harel, the expressive power of relational query languages can be represented by set diagrams. Figure 1 shows such a set diagram where the following query languages are represented as sets of partial functions: The first-order query language [12], Data$\log$ [3], Datalog with stratified negation, and QPTIME, i.e. the set of relational queries that are evaluable in polynomial time [10]. For every atomic subset, a typical query is indicated. These queries are described below.

ForAll: Let $\mathcal{G}$ be any finite graph. Query: Given a vertex $v$, is there an edge from $v$ to $v^{\prime}$ for all vertices $v^{\prime}$ ?

Exists: Let $\mathcal{G}$ be any finite graph. Query: Given a vertex $v$, does at least one edge exist from $v$ to any vertex $v^{\prime}$ ?

TC (Transitive Closure): Let $\mathcal{G}$ be any finite graph. Query: Given a vertex $v$, which are the vertices $v^{\prime}$ that are reachable from $v$ (i.e. there is a directed path from $v$ to $v^{\prime}$ )?

NotTC: Let $\mathcal{G}$ be any finite graph. Query: Given a vertex $v$, which are the vertices $v^{\prime}$ that are not reachable from $v$ (i.e. there is no directed path from $v$ to $\left.v^{\prime}\right)$ ?

Game: Let $\mathcal{G}$ be any rooted tree where every leaf node is either black or white. There are two players who know $\mathcal{G}$ and the color of the leaf nodes. The root node is the start position of the game. Player I starts moving down the tree by one edge. Then, player II moves down another edge. They continue until a leaf node is reached. Player I wins if the leaf node is black and player II wins if the leaf node is white. Query: Does player I have a winning 


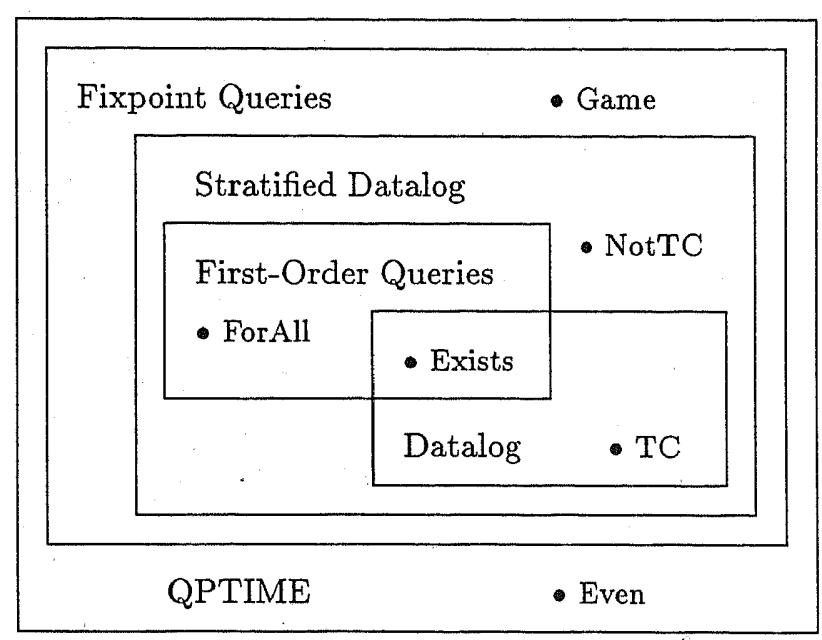

Figure 1: Expressive power of relational query languages according to Chandra and Harel.

strategy? Or equivalently, can player I win independently of the moves by player II?

Even: Let $\mathcal{G}$ be any finite graph. Query: Is the number of vertices an even number?

In Chandra and Harel's approach [10], a relational database $(D, \bar{R})$ of the type $\bar{a}=\left(a_{0}, \ldots, a_{k-1}\right)$ consists of a domain $D$ and a $k$-tuple $\bar{R}=\left(R_{0}, \ldots, R_{k-1}\right)$ where $R_{i} \subseteq D^{a_{i}}$. The domain $D$ is assumed to be a finite subset of a countable universe $U$. Chandra and Harel restrict themselves to queries of type $(\bar{a}, b)$, i.e. queries $Q$ mapping a relational database $(D, \bar{R})$ of type $\bar{a}$ to a $b$-ary relation $Q(D, \bar{R}) \subseteq D^{b}$. From this restriction follows that there is no query to express the bill of materials as shown in the following. Let $D=\{0, \ldots, 2 n-1\}$ be the domain and let $R_{0}$ be the relation defining the part/subpart structure shown in Figure 2. The parts are identified uniquely by numbers such that $D=\left\{p_{1}, \ldots, p_{n-1}, q_{1}, \ldots, q_{n-1}, r, s\right\}$. We define the following costs:

$$
\begin{aligned}
& \operatorname{cost}(r)=0 \\
& \operatorname{cost}(s)=1
\end{aligned}
$$




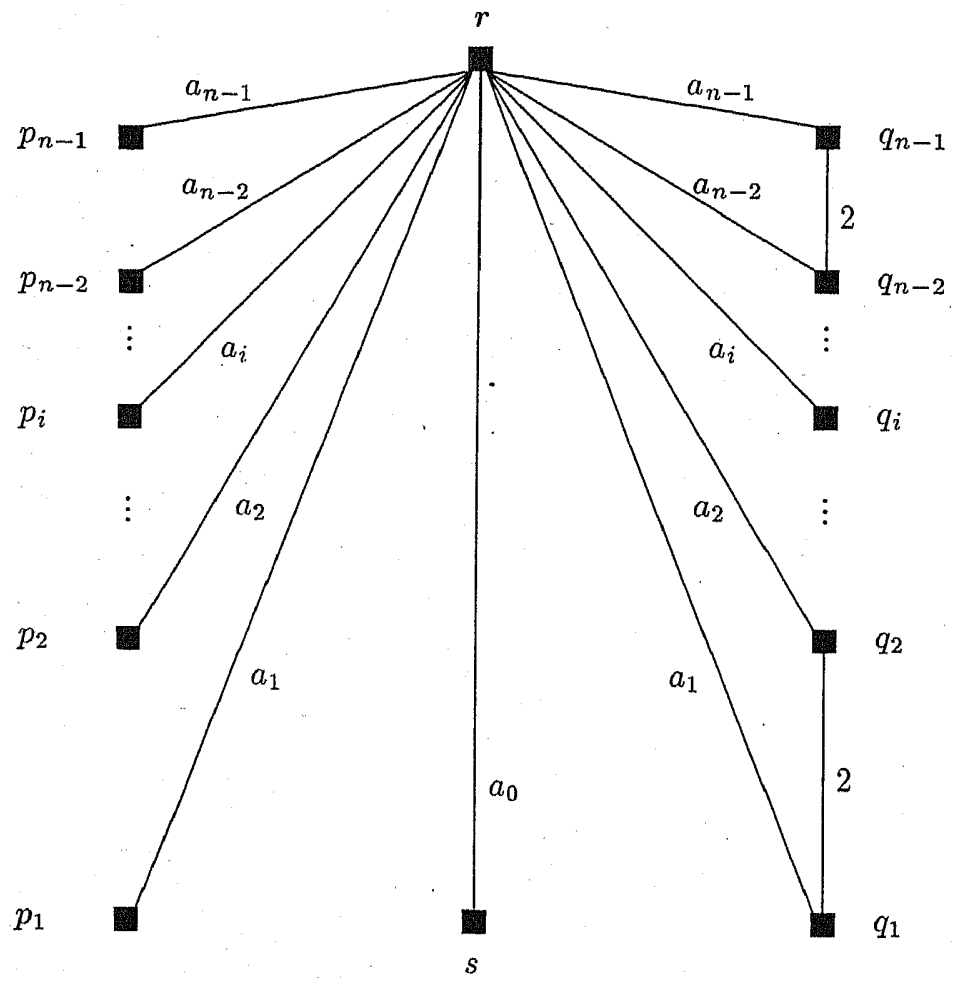

Figure 2: Given this part/subpart structure, the total cost of part $r$ and its subparts cannot be expressed by any query considered by Chandra and Harel. 


$$
\begin{array}{lll}
\operatorname{cost}\left(p_{i}\right)=1 & & 0<i<n \\
\operatorname{cost}\left(q_{i}\right)=1 & & 0<i<n
\end{array}
$$

The part $q_{i}$ is contained twice in $q_{i-1}$. The parts $p_{i}$ and $q_{i}$ are contained $a_{i}$ times in $r$ where $a_{i} \in\{0,1\}$. Similarly, the part $s$ is contained $a_{0}$ times in $r$. The cost of $r$ and all its subparts are

$$
\text { total_cost }(r)=\sum_{i=0}^{n-1} a_{i} 2^{i}
$$

as it can easily be shown by induction on $n$. Thus, depending on the part/subpart structure, i.e. depending on $a_{0}, \ldots, a_{n-1}$, the cost are

$$
\text { total_cost }(r) \in\left\{0, \ldots, 2^{n}-1\right\}
$$

and hence, the domain $D=\{0, \ldots, 2 n-1\}$ does not contain enough elements to represent every possible answer if $n>2$. More generally speaking, query languages having the ability to count cannot be analyzed within the framework of [10].

In this paper, we present a more general framework to study the expressive power of arbitrary query languages. We rely on an algebraic approach where heterogeneous structures represent information systems and morphisms represent the correspondences between the instances of the databases, the correspondences between the answers, and the correspondences between the queries. Let $I S_{1}$ and $I S_{2}$ be two structures, each representing an information system. Such an information system consists of a query language, a set of database instances, a set of answers, and a query evaluation function. We call the query language of $I S_{2}$ as expressive as the query language of $I S_{1}$ iff there exists a morphism from $I S_{1}$ to $I S_{2}$. In Chandra and Harel's approach "as expressive as" is defined by means of set inclusion whereas in our approach, "as expressive as" is defined by means of morphisms. This kind of generalization is often used in mathematics. In our case, this generalization facilitates the comparison of the expressive power of query languages of different types. This more general comparison capability is demonstrated by comparing a Boolean query language and a weighted query language consisting of vague queries. The answer to a Boolean query consists of a set of data items, whereas the answer to a weighted query consists of a function which assigns every data item a probability that the data item belongs to the answer. We shall present 
a necessary and sufficient condition for the cases where the Boolean query language is less expressive than the weighted query language.

A further generalization consists of allowing infinite domains. It should be noted that infinite domains are not only important for counting but also for vague queries [15], [23] and for Information Retrieval query languages [22], [27]. Infinite domains do not necessarily lead to unbounded computation times. This fact is demonstrated by a query language FQL* which is more expressive than the fixpoint query languages but still evaluable in polynomial time.

FQL* is a Functional Query Language with a syntax similar to the syntax of OSQL [6]. OSQL is an interactive interface to the Iris DBMS [28]. Unlike the specifications of OSQL functions, the specifications of FQL* functions can be recursive or even mutually recursive. FQL* has a fixpoint semantics based on a complete lattice consisting of bag functions. Examples of recursive FQL* functions are given that determine the ancestors of persons and the bill of materials. It is shown that these $\mathrm{FQL}^{*}$ functions are computed in polynomial time even if the parents relation and the parts_of relation contain cycles. In these cases, a person may be an ancestor of himself and the cost of a part may be infinite. We show that there are non-hierarchical relations where these results are meaningful.

The main contributions of this paper are the following. First of all, a new notion of expressive power is introduced which facilitates the comparison of formal languages with respect to their expressive power even when these query languages and their underlying data model are of different types. Second, a new criterion is presented which characterizes the cases when a Boolean query language is less expressive than a weighted query language. Finally, an object-oriented query language is introduced which has an expressive power that goes beyond the expressive power of the fixpoint query languages.

The paper is organized as follows. In Section 2, our new notion of expressive power is introduced. In Section 3, we show that for relational query languages, Chandra and Harel's notion of expressive power coincides with our notion of expressive power. In Section 4, the expressive power of a Boolean query language and the expressive power of a weighted query language are compared. In Section 5 , the object-oriented query language $\mathrm{FQL}^{*}$ is introduced. In Section 6 , we discuss the expressive power of FQL*. In Section 7, some conclusions are drawn. Appendix A contains the proofs of two theorems. 


\section{A New Notion of Expressive Power}

In this section, we introduce a new notion of expressive power. We start with a simple model of an information system. An information system is represented by a structure, i.e. a heterogeneous algebra [7]

$$
I S:=\langle D B S, Q L, A S, \alpha\rangle
$$

where the database system, $D B S$, is the set of possible instances of the database; the query language, $Q L$, is the set of possible queries; the answer system, $A S$, is the set of possible answers; the query evaluation function, $\alpha$, is a partial function mapping $D B S \times Q L$ to $A S$. The following example shows an information system based on a relational database system that is accessible by means of relational algebra.

Example 1 The components of the information system

$$
I S_{r a}=\left\langle D B S_{r e l}, Q L_{r a}, A S_{r e l}, \alpha_{r a}\right\rangle
$$

are the following. The database system $D B S_{\text {rel }}$ consists of all instances of a relational database. Every instance $(D, \bar{R})$ is represented by a domain $D$ and tuple of relations $\bar{R}=\left(R_{0}, \ldots, R_{k-1}\right)$ where $R_{i} \subseteq D^{a_{i}}$. The query language $Q L_{r a}$ consists of relational algebra expressions [26]. The answer system $A S_{\text {rel }}$ consists of relations $R \subseteq D^{b}$. The evaluation function $\alpha_{r a}$ assigns every instance $(D, \bar{R})$ of the database and every query $q$ an answer $\alpha_{r a}((D, \bar{R}), q)$.

A complete specification of an information system should also contain the specification of a modification language $M L$ and the specification of a modification function $\mu: D B S \times M L \rightarrow D B S$. Since, in this paper, we are interested only in the expressive power of the query language, it is sufficient to represent an information system by the simplified structure (1) which defines neither a modification language nor a modification function.

Below, we define when a query language $Q L_{2}$ is as expressive as a query language $Q L_{1}$. We will see that this relationship between $Q L_{1}$ and $Q L_{2}$ depends on (1) the information systems to which $Q L_{1}$ and $Q L_{2}$ belong, (2) the correspondences between the instances $d b_{1} \in D B S_{1}$ and the instances $d b_{2} \in D B S_{2}$, and (3) the correspondences between the answers $a_{1} \in A S_{1}$ and the answers $a_{2} \in A S_{2}$. The correspondences between the instances of the databases are given by the function $f$, the correspondences between the answers are given 


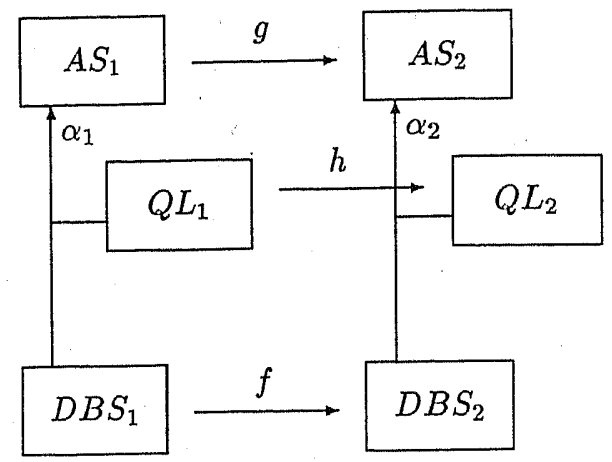

Figure 3: Expressive power of query languages.

by the function $g$, and the correspondences between the queries are represented by the function $h$ (Figure 3 ).

Definition 1 Let $I S_{1}=\left\langle D B S_{1}, Q L_{1}, A S_{1}, \alpha_{1}\right\rangle$ and $I S_{2}=\left\langle D B S_{2}\right.$, $\left.Q L_{2}, A S_{2}, \alpha_{2}\right\rangle$ be two information systems and let $f: D B S_{1} \rightarrow D B S_{2}$ be a function which determines for every instance $d b_{1} \in D B S_{1}$ a corresponding instance $f\left(d b_{1}\right) \in D B S_{2}$ and let $g: \operatorname{range}\left(\alpha_{1}\right) \rightarrow$ range $\left(\alpha_{2}\right)$ be an injective function which determines for every answer $a_{1} \in$ range $\left(\alpha_{1}\right)$ a corresponding answer $g\left(a_{1}\right) \in \operatorname{range}\left(\alpha_{2}\right)$. The query language $Q L_{2}$ of $I S_{2}$ is called as expressive as the query language $Q L_{1}$ of $I S_{1}$ with respect to the correspondences given by $f$ and $g$ iff there exists a function $h: Q L_{1} \rightarrow Q L_{2}$ such that

$$
\forall\left(d b_{1}, q_{1}\right) \in \operatorname{dom}\left(\alpha_{1}\right): g\left(\alpha_{1}\left(d b_{1}, q_{1}\right)\right)=\alpha_{2}\left(f\left(d b_{1}\right), h\left(q_{1}\right)\right) .
$$

The function $g$ is required to be injective because two different answers of $A S_{1}$ must correspond to two different answers of $A S_{2}$. The function $f$ is not required to be injective because several equivalent instances in $D B S_{1}$ may correspond to a single instance in $D B S_{2}$ (see below). Subsequently,

$$
Q L_{1}\left(I S_{1}\right) \quad \leq_{f, g} \quad Q L_{2}\left(I S_{2}\right)
$$

denotes that $Q L_{2}$ of $I S_{2}$ is as expressive as $Q L_{1}$ of $I S_{1}$ with respect to the correspondences given by $f$ and $g$. The relationship $\leq_{f, g}$ can also be characterized by means of morphisms. Morphisms are defined as follows. 
Definition 2 Let $\mathcal{S}=\langle U, V, W, \alpha\rangle$ and $\mathcal{T}=\langle X, Y, Z, \beta\rangle$ be two structures where $\alpha: U \times V \rightarrow W$ and $\beta: X \times Y \rightarrow Z$ are partial functions. The set of functions $\{f: U \rightarrow X, g: W \rightarrow Z, h: V \rightarrow Y\}$ is called a morphism if

$$
\forall(u, v) \in \operatorname{dom}(\alpha): g(\alpha(u, v))=\beta(f(u), h(v))
$$

If the functions $f, g$, and $h$ are bijective the morphism $\{f, g, h\}$ is called an isomorphism [7].

Proposition 1 Let $I S_{1}=\left\langle D B S_{1}, Q L_{1}, A S_{1}, \alpha_{1}\right\rangle$ and $I S_{2}=\left\langle D B S_{2}\right.$, $\left.Q L_{2}, A S_{2}, \alpha_{2}\right\rangle$ be two information systems and let $f: D B S_{1} \rightarrow D B S_{2}$ be a function which determines for every instance $d b_{1} \in D B S_{1}$ a corresponding instance $f\left(d b_{1}\right) \in D B S_{2}$ and let $g: \operatorname{range}\left(\alpha_{1}\right) \rightarrow$ range $\left(\alpha_{2}\right)$ be an injective function which determines for every answer $a_{1} \in$ range $\left(\alpha_{1}\right)$ a corresponding answer $g\left(a_{1}\right) \in$ range $\left(\alpha_{2}\right)$. The query language $Q L_{2}$ of $I S_{2}$ is as expressive as the query language $Q L_{1}$ of $I S_{1}$ with respect to the correspondences given by $f$ and $g$ iff there exists a function $h: Q L_{1} \rightarrow Q L_{2}$ such that $\left\{f: D B S_{1} \rightarrow D B S_{2}, g\right.$ : range $\left.\left(\alpha_{1}\right) \rightarrow \operatorname{range}\left(\alpha_{2}\right), h: Q L_{1} \rightarrow Q L_{2}\right\}$ is a morphism from $I S_{1}$ to $I S_{2}$.

Proof. $\{f, g, h\}$ is a morphism because of (2). Conversely, (2) is satisfied because $\{f, g, h\}$ is a morphism. $\diamond$

In order to define "equally expressive", we introduce an equivalence relation between the instances of the database and an equivalence relation between the queries. Given an information system $I S=\langle D B S, Q L, A S, \alpha\rangle$, two instances $d b, d b^{\prime} \in D B S$ are called equivalent iff for every query $q \in Q L$, the answer $\alpha(d b, q)$ is equal to the answer $\alpha\left(d b^{\prime}, q\right)$. Similarly, two queries $q, q^{\prime} \in Q L$ are called equivalent iff for every instance $d b \in D B S$, the answer $\alpha(d b, q)$ is equal to the answer $\alpha\left(d b, q^{\prime}\right)$.

$$
\begin{aligned}
d b \approx d b^{\prime} & : \Longleftrightarrow \forall q \in Q L: \alpha(d b, q)=\alpha\left(d b^{\prime}, q\right) \\
q \approx q^{\prime} & : \Longleftrightarrow \forall d b \in D B S: \alpha(d b, q)=\alpha\left(d b, q^{\prime}\right)
\end{aligned}
$$

Example 2 Let $\left\langle D B S_{r e l}, Q L_{r a}, A S_{r e l}, \alpha_{r a}\right\rangle$ be an information system consisting of a relational database system accessible through relational algebra (Example 1). The following queries are equivalent.

$$
\begin{aligned}
& \text { select }[\text { age }=20](\text { project }[\text { name, age }](\text { employee })) \\
& \text { project }[\text { name, age }](\text { select }[\text { age }=20](\text { employee }))
\end{aligned}
$$


It is easy to show that both the relation $\approx$ defined on $D B S$ and the relation $\approx$ defined on $Q L$ are equivalence relations, i.e. reflexive, symmetric, and transitive relations. Hence, the set $D B S$ and the set $Q L$ are partitioned into disjoint equivalence classes. The class consisting of all instances that are equivalent to the instance $d b$ is denoted by $[d b]$. Similarly, the class consisting of all queries that are equivalent to the query $q$ is denoted by $[q]$. The set of all equivalence classes of instances in $D B S$ is denoted by $D B S^{\approx}$ and the set of all equivalence classes of queries of $Q L$ is denoted by $Q L \approx$. Using this notation, every evaluation function $\alpha$ determines a function $\alpha \approx$.

$$
\alpha \approx: D B S^{\approx} \times Q L^{\approx} \rightarrow A S,([d b],[q]) \mapsto \alpha(d b, q)
$$

Note that $\alpha \approx$ is well defined. From $d b \approx d b^{\prime}$ and $q \approx q^{\prime}$ follows that $\alpha(d b, q)=\alpha\left(d b^{\prime}, q^{\prime}\right)$ and hence, the function value $\alpha \approx([d b],[q])$ is independent from the particular instance $d b$ representing $[d b]$ and from the particular query $q$ representing $[q]$. Every function $f: D B S_{1} \rightarrow$ $D B S_{2}$ satisfying

$$
d b_{1} \approx d b_{1}^{\prime} \Longrightarrow f\left(d b_{1}\right) \approx f\left(d b_{1}^{\prime}\right)
$$

induces a mapping $f^{\approx}$ from $D B S_{1}^{\approx}$ to $D B S_{2}^{\approx}$.

$$
f^{\approx}: D B S_{1}^{\approx} \rightarrow D B S_{2}^{\approx},\left[d b_{1}\right] \mapsto\left[f\left(d b_{1}\right)\right]
$$

Note that the function value $f \approx\left(\left[d b_{1}\right]\right)$ is well defined. Because of (8) it is independent of the particular instance $d b_{1}$ representing the equivalence class $\left[d b_{1}\right]$.

The relationship "equally expressive" can be defined in two different ways. First, it can be defined by means of an isomorphisms from $\left\langle D B S_{1}^{\approx}, Q L_{1}^{\approx}, A S_{1}, \alpha_{1}^{\approx}\right\rangle$ to $\left\langle D B S_{2}^{\approx}, Q L_{2}^{\approx}, A S_{2}, \alpha_{2} \approx\right\rangle$. Second, it can be defined by means of $\leq f, g$ and $\geq_{f^{*}, g^{-1}}$ provided the correspondences given by $f: D B S_{1} \rightarrow D B S_{2}$ and the correspondences given by $f^{*}: D B S_{2} \rightarrow D B S_{1}$ are compatible (i.e. $f^{*}\left(f\left(d b_{1}\right)\right) \approx d b_{1}$ and $\left.f\left(f^{*}\left(d b_{2}\right)\right) \approx d b_{2}\right)$. Figure 4 shows that this kind of compatibility requires a bijective correspondence between the equivalence classes rather than between the particular instances of the two databases. For instance, the equivalence class $\left\{d b_{1}, d b_{1}^{\prime}, d b_{1}^{\prime \prime}\right\}$ corresponds to the class $\left\{d b_{2}, d b_{2}^{\prime}\right\}$ and there exists no bijective correspondence between the elements of the classes.

We will use isomorphisms, i.e. the first alternative, to define the relationship "equally expressive." Later, we will show that this def- 


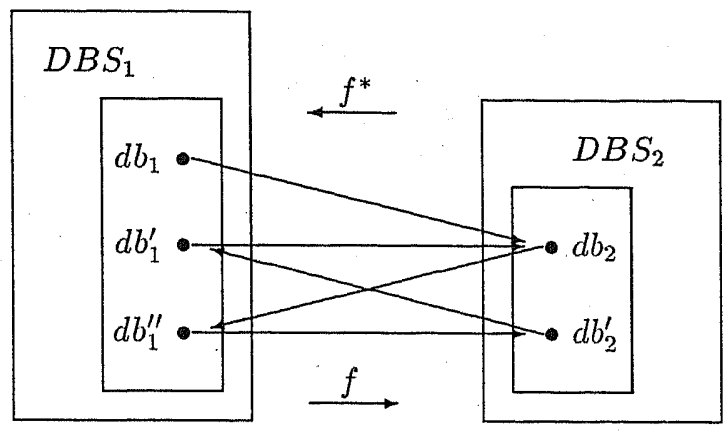

Figure 4: Correspondences between equivalence classes.

inition is equivalent to the second alternative (Proposition 4). This shows that the concept of "as expressive as" is quite a robust concept.

Definition 3 Let $I S_{1}=\left\langle D B S_{1}, Q L_{1}, A S_{1}, \alpha_{1}\right\rangle$ and $I S_{2}=\left\langle D B S_{2}\right.$, $\left.Q L_{2}, A S_{2}, \alpha_{2}\right\rangle$ be two information systems. Assume that the correspondences between the instances of the databases and between the answers are given by the function $f: D B S_{1} \rightarrow D B S_{2}$ and by the function $g: A S_{1} \rightarrow A S_{2}$ respectively. $Q L_{1}$ of $I S_{1}$ and $Q L_{2}$ of $I S_{2}$ are called equally expressive with respect to $f$ and $g$, denoted by

$$
Q L_{1}\left(I S_{1}\right)==_{f, g} \quad Q L_{2}\left(I S_{2}\right),
$$

iff $f$ satisfies (8) and there exists a function $h \approx: Q L_{1} \approx \rightarrow Q Q \approx$ such that the morphism $\{f \approx, g, h \approx\}$ is an isomorphism from $\left\langle D B S_{1} \approx, Q L_{1}^{\approx}\right.$, $\left.A S_{1}, \alpha \approx \widetilde{1}\right\rangle$ to $\left\langle D B S_{2} \approx, Q L \widetilde{2}, A S_{2}, \alpha \widetilde{2}\right\rangle$.

The existence of an isomorphism $\{f, g, h\}$ from the information system $\left\langle D B S_{1}, Q L_{1}, A S_{1}, \alpha_{1}\right\rangle$ to the information system $\left\langle D B S_{2}, Q L_{2}\right.$, $\left.A S_{2}, \alpha_{2}\right\rangle$ implies the existence of an isomorphism $\{f \approx, g, h \approx\}$ from $\left\langle D B S_{1} \approx, Q L_{1}^{\approx}, A S_{1}, \alpha \widetilde{1}\right\rangle$ to $\left\langle D B S_{2} \widetilde{2}, Q L_{2}, A S_{2}, \alpha \widetilde{2}\right\rangle$ but not vice versa. This asymmetry is because two equivalent instances $d b_{1}$ and $d b_{1}^{\prime}$ in $D B S_{1}$ may correspond to a single instance $d b_{2}$ in $D B S_{2}$ (see Figure 4) or two equivalent queries $q_{1}$ and $q_{1}^{\prime}$ in $Q L_{1}$ may correspond to a single query $q_{2}$ in $Q L_{2}$. Thus, two query languages can be equally expressive even if there are no bijective correspondences between the queries or between the instances of the databases. 
Definition $4 Q L_{2}$ of $I S_{2}$ is more expressive than $Q L_{1}$ of $I S_{1}$ with respect to $f$ and $g$, denoted by

$$
Q L_{1}\left(I S_{1}\right)<_{f, g} \quad Q L_{2}\left(I S_{2}\right)
$$

iff $Q L_{2}$ is as expressive as $Q L_{1}$ but $Q L_{1}$ and $Q L_{2}$ are not equally expressive.

The following proposition will be useful in the subsequent sections where we will prove that some query languages are more expressive than others.

Proposition 2 If $Q L_{1}\left(I S_{1}\right)={ }_{f, g} Q L_{2}\left(I S_{2}\right)$, there exists for every query $q_{2} \in Q L_{2}$ a query $q_{1} \in Q L_{1}$ such that for all $d b_{1} \in D B S_{1}$,

$$
\left(d b_{1}, q_{1}\right) \in \operatorname{domain}\left(\dot{\alpha}_{1}\right) \Rightarrow g\left(\alpha_{1}\left(d b_{1}, q_{1}\right)\right)=\alpha_{2}\left(f\left(d b_{1}\right), q_{2}\right)
$$

Proof. From $Q L_{1}\left(I S_{1}\right)={ }_{f, g} Q L_{2}\left(I S_{2}\right)$ it follows that every query $q_{2}$ belongs to an equivalence class $\left[h\left(q_{1}\right)\right]$. From (4) follows that $q_{1}$ satisfies (12). $\diamond$

If $Q L_{1}\left(I S_{1}\right) \leq_{f, g} Q L_{2}\left(I S_{2}\right)$ and we want to prove that $Q L_{1}\left(I S_{1}\right)$ $<_{f, g} Q L_{2}\left(I S_{2}\right)$, it is sufficient to show that (12) yields a contradiction. The following proposition shows the transitivity of the relationships $\leq_{f, g},=_{f, g}$, and $<_{f, g}$.

Proposition 3 Given $\theta \in\{\leq,=,<\}$, from $Q L_{1}\left(I S_{1}\right) \theta_{f, g} Q L_{2}\left(I S_{2}\right)$ and $Q L_{2}\left(I S_{2}\right) \theta_{f^{\prime}, g^{\prime}} Q L_{3}\left(I S_{3}\right)$ follows $Q L_{1}\left(I S_{1}\right) \theta_{f^{\prime} \circ f, g^{\prime} \circ g} Q L_{3}\left(I S_{3}\right)$.

Proof. The transitivity of $\leq_{f, g},=_{f, g}$, and $<_{f, g}$ follows immediately from their definitions. $\diamond$

Finally, we will show that $=_{f, g}$ is equivalent to $\leq_{f, g}$ and $\geq_{f^{*}, g-1}$ provided the correspondences given by $f$ and the correspondences given by $f^{*}$ are compatible.

Proposition 4 Let $I S_{1}=\left\langle D B S_{1}, Q L_{1}, A S_{1}, \alpha_{1}\right\rangle$ and $I S_{2}=\left\langle D B S_{2}\right.$, $\left.Q L_{2}, A S_{2}, \alpha_{2}\right\rangle$ be two information systems. Assume that the correspondences between the instances of the databases and between the answers are given by the function $f: D B S_{1} \rightarrow D B S_{2}$ and by the function $g: A S_{1} \rightarrow A S_{2}$ respectively. Then, $Q L_{1}\left(I S_{1}\right)=_{f, g} Q L_{2}\left(I S_{2}\right)$ iff there is a function $f^{*}: D B S_{2} \rightarrow D B S_{1}$ compatible with $f$ (i.e. $\forall d b_{2} \in$ $D B S_{2}: f\left(f^{*}\left(d b_{2}\right)\right) \approx d b_{2}$ and $\left.\forall \dot{d} b_{1} \in D B S_{1}: f^{*}\left(f\left(d b_{1}\right)\right) \approx d b_{1}\right)$ such that $Q L_{1}\left(I S_{1}\right) \leq_{f, g} Q L_{2}\left(I S_{2}\right)$ and $Q L_{1}\left(I S_{1}\right) \geq_{f^{*}, g^{-1}} Q L_{2}\left(I S_{2}\right)$. 
Proof. From $Q L_{1}\left(I S_{1}\right)=f_{f, g} Q L_{2}\left(I S_{2}\right)$ it follows that $Q L_{1}\left(I S_{1}\right) \leq_{f, g}$ $Q L_{2}\left(I S_{2}\right)$ because for every $\left(d b_{1}, q_{1}\right) \in \operatorname{range}\left(\alpha_{1}\right)$,

$$
\begin{aligned}
g\left(\alpha_{1}\left(d b_{1}, q_{1}\right)\right) & =g\left(\alpha_{\tilde{I}}\left(\left[d b_{1}\right],\left[q_{1}\right]\right)\right) \\
& =\alpha_{\tilde{2}}\left(f \approx\left(\left[d b_{1}\right]\right), h \approx\left(\left[q_{1}\right]\right)\right) \\
& =\alpha_{2}\left(f\left(d b_{1}\right), h\left(q_{1}\right)\right) .
\end{aligned}
$$

Assume that $Q L_{1}\left(I S_{1}\right)={ }_{f, g} Q L_{2}\left(I S_{2}\right)$. Since $\{f \approx, g, h \approx\}$ is an isomorphism, there exists a function $f^{*}: D B S_{2} \rightarrow D B S_{1}$ such that $\forall d b_{2} \in D B S_{2}: f\left(f^{*}\left(d b_{2}\right)\right) \approx d b_{2}$ and $\forall d b_{1} \in D B S_{1}: f^{*}\left(f\left(d b_{1}\right)\right) \approx d b_{1}$ (see also Figure 4). For the same reasons, there exists a function $h^{*}: Q L_{2} \rightarrow Q L_{1}$ such that $\forall q_{2} \in Q L_{2}: h\left(h^{*}\left(q_{2}\right)\right) \approx q_{2}$ and $\forall q_{1} \in$ $Q L_{1}: h^{*}\left(h\left(q_{1}\right)\right) \approx q_{1}$. For every $\left(d b_{2}, q_{2}\right) \in \operatorname{range}\left(\alpha_{2}\right)$, we obtain the equalities

$$
\begin{aligned}
g\left(\alpha_{1}\left(f^{*}\left(d b_{2}\right), h^{*}\left(q_{2}\right)\right)\right) & =g\left(\alpha_{1}^{\approx}\left(\left[f^{*}\left(d b_{2}\right)\right],\left[h^{*}\left(q_{2}\right)\right]\right)\right) \\
& =\alpha_{2}^{\approx}\left(f^{\approx}\left(\left[f^{*}\left(d b_{2}\right)\right]\right), h^{\approx}\left(\left[h^{*}\left(q_{2}\right)\right]\right)\right) \\
& =\alpha_{2}\left(f\left(f^{*}\left(d b_{2}\right)\right), h\left(h^{*}\left(q_{2}\right)\right)\right) \\
& =\alpha_{2}\left(d b_{2}, q_{2}\right) .
\end{aligned}
$$

Since $g$ is bijective, $\alpha_{1}\left(f^{*}\left(d b_{2}\right), h^{*}\left(q_{2}\right)\right)=g^{-1}\left(\alpha_{2}\left(d b_{2}, q_{2}\right)\right)$ and hence, $Q L_{1}\left(I S_{1}\right) \geq_{f^{*}, g^{-1}} Q L_{2}\left(I S_{2}\right)$.

The other direction is proven as follows. Assume that there exists a function $f^{*}: D B S_{2} \rightarrow D B S_{1}$ such that $\forall d b_{2} \in D B S_{2}: f\left(f^{*}\left(d b_{2}\right)\right) \approx$ $d b_{2}, \forall d b_{1} \in D B S_{1}: f^{*}\left(f\left(d b_{1}\right)\right) \approx d b_{1}, Q L_{1}\left(I S_{1}\right) \leq_{f, g} Q L_{2}\left(I S_{2}\right)$, and $Q L_{1}\left(I S_{1}\right) \geq_{f^{*}, g^{-1}} Q L_{2}\left(I S_{2}\right)$. The function $f \approx$ is well defined because

$$
\begin{aligned}
& f\left(d b_{1}\right) \not f f\left(d b_{1}^{\prime}\right) \\
& \quad \Longrightarrow \exists q_{2}: \alpha_{2}\left(f\left(d b_{1}\right), q_{2}\right) \neq \alpha_{2}\left(f\left(d b_{1}^{\prime}\right), q_{2}\right) \\
& \quad \Longrightarrow \exists q_{2}: g^{-1}\left(\alpha_{2}^{\circ}\left(f\left(d b_{1}\right), q_{2}\right)\right) \neq g^{-1}\left(\alpha_{2}\left(f\left(d b_{1}^{\prime}\right), q_{2}\right)\right) \\
& \quad \Longrightarrow \exists q_{2}: \alpha_{1}\left(f^{*}\left(f\left(d b_{1}\right)\right), h^{*}\left(q_{2}\right)\right) \neq \alpha_{1}\left(f^{*}\left(f\left(d b_{1}^{\prime}\right)\right), h^{*}\left(q_{2}\right)\right) \\
& \quad \Longrightarrow \exists q_{1}: \alpha_{1}\left(d b_{1}, q_{1}\right) \neq \alpha_{1}\left(d b_{1}^{\prime}, q_{1}\right) \\
& \quad \Longrightarrow d b_{1} \not d d b_{1}^{\prime} .
\end{aligned}
$$

This implication is equivalent to (8). The function $f \approx$ is injective because

$$
d b_{1} \not \approx d b_{1}^{\prime} \Longrightarrow \exists q_{1}: \alpha_{1}\left(d b_{1}, q_{1}\right) \neq \alpha_{1}\left(d b_{1}^{\prime}, q_{1}\right)
$$




$$
\begin{aligned}
& \Longrightarrow \exists q_{1}: g\left(\alpha_{1}\left(d b_{1}, q_{1}\right)\right) \neq g\left(\alpha_{1}\left(d b_{1}^{\prime}, q_{1}\right)\right) \\
& \left.\left.\Longrightarrow \exists q_{1}: \alpha_{2}\left(f\left(d b_{1}\right), h\left(q_{1}\right)\right)\right) \neq \alpha_{2}\left(f\left(d b_{1}^{\prime}\right), h\left(q_{1}\right)\right)\right) \\
& \Longrightarrow f\left(d b_{1}\right) \not f f\left(d b_{1}^{\prime}\right) .
\end{aligned}
$$

The function $f^{\approx}$ is surjective because for $\forall d b_{2} \in D B S_{2}: f\left(f^{*}\left(d b_{2}\right)\right) \approx$ $d b_{2}$. This shows that $f \approx$ is bijective.

Since $g^{-1}$ exists, the function $g$ is bijective. The function $h \approx$ is well defined because

$$
\begin{aligned}
q_{1} \approx q_{1}^{\prime} & \Longleftrightarrow \forall d b_{1}: \alpha_{1}\left(d b_{1}, q_{1}\right)=\alpha_{1}\left(d b_{1}, q_{1}^{\prime}\right) \\
& \Longleftrightarrow \forall d b_{1}: g\left(\alpha_{1}\left(d b_{1}, q_{1}\right)\right)=g\left(\alpha_{1}\left(d b_{1}, q_{1}^{\prime}\right)\right) \\
& \Longleftrightarrow \forall d b_{1}: \alpha_{2}\left(f\left(d b_{1}\right), h\left(q_{1}\right)\right)=\alpha_{2}\left(f\left(d b_{1}\right), h\left(q_{1}^{\prime}\right)\right) \\
& \Longleftrightarrow \forall d b_{2}: \alpha_{2}\left(d b_{2}, h\left(q_{1}\right)\right)=\alpha_{2}\left(d b_{2}, h\left(q_{1}^{\prime}\right)\right) \\
& \Longleftrightarrow h\left(q_{1}\right) \approx h\left(q_{1}^{\prime}\right) .
\end{aligned}
$$

We used the fact that $g$ is injective and $f \approx$ is surjective. At the same time, we can conclude that $h \approx$ is injective.

The function $h \approx$ is surjective because $q_{2} \in Q L_{2}$, we have for every $d b_{2} \in D B S_{2}$ :

$$
\begin{aligned}
\alpha_{2}\left(d b_{2}, q_{2}\right) & =g\left(\alpha_{1}\left(f^{*}\left(d b_{2}\right), h^{*}\left(q_{2}\right)\right)\right) \\
& =\alpha_{2}\left(f\left(f^{*}\left(d b_{2}\right)\right), h\left(h^{*}\left(q_{2}\right)\right)\right) \\
& =\alpha_{2} \dot{(}\left(d b_{2}, h\left(h^{*}\left(q_{2}\right)\right)\right) .
\end{aligned}
$$

and hence, $h\left(h^{*}\left(q_{2}\right)\right) \approx q_{2}$. This' shows that $h^{\approx}$ is bijective.

Because $\{f, g, h\}$ is a morphism, $\{f \approx, g, h \approx\}$ is also a morphism. It is actually an isomorphism because $f \approx, g$, and $h \approx$ are bijective and hence, $Q L_{1}\left(I S_{1}\right)={ }_{f, g} Q L_{2}\left(I S_{2}\right)$. $\diamond$

In this section, we have defined "as expressive as" $\left(\leq_{f, g}\right)$, "equally expressive" $\left(=_{f, g}\right)$, and "more expressive than" $\left(<_{f, g}\right)$. The first relationship is associated with a morphism from one information system to another information system. The second relationship is associated with an isomorphism from the first information system modulo $\approx$ to the second information system modulo $\approx$. Alternatively, $=_{f, g}$ can be defined as $\leq_{f, g}$ and $\geq_{f^{*}, g-1}$. The third relationship is defined as usual, i.e. as $\leq_{f, g}$ and $\neq_{f, g}$. In the subsequent sections, such relationships will be identified between query languages of various types. 


\section{Relational Query Languages}

In this section, we first define what relational query languages are, in the sense of Chandra and Harel [9], [10]. We will show that when comparing the expressive power of relational query languages, Chandra and Harel's notion of expressive power is equivalent to our notion of expressive power.

An instance $(D, \bar{R})$ of a relational database system $D B S_{\text {rel }}$ consists of a finite domain $D$ and relations $\bar{R}=\left(R_{0}, \ldots, R_{k-1}\right)$ where $R_{i} \subseteq$ $D^{a_{i}}$. The countable universe $U$ is assumed to entail all domains.

$$
\forall(D, \bar{R}) \in D B S_{r e l}: D \subseteq U \wedge|D| \in \mathrm{N}
$$

The answer system contains all relations that have a finite arity.

$$
A S_{r e l}:=\left\{R \subseteq U^{b} \mid b \in N\right\}
$$

Definition 5 (Chandra \& Harel) Given an information system IS $=\left\langle D B S_{r e l}, Q L, A S_{r e l}, \alpha\right\rangle$, the query language $Q L$ is called relational iff for every query $q \in Q L$, there exist a tuple $\bar{a}=\left(a_{0}, \ldots, a_{k-1}\right) \in \mathrm{N}^{k}$ and $a$ natural number $b \in \mathrm{N}$ such that from $((D, \bar{R}), q) \in \operatorname{dom}(\alpha)$ it follows that $\bar{R}=\left(R_{0}, \ldots, R_{k-1}\right)$ where $R_{i} \subseteq D^{a_{i}}$ and $\alpha((D, \bar{R}), q) \subseteq$ $D^{b}$. The tuple $(\bar{a}, b)$ is called the type of the query $q$. The query $q$ is called computable iff the function

$$
F_{q}: D B S_{r e l} \rightarrow A S_{r e l},(D, \bar{R}) \mapsto \alpha((D, \bar{R}), q)
$$

is a partial recursive function and for every isomorphism $h$ from $(D, \bar{R})$ to $\left(D^{\prime}, \bar{R}^{\prime}\right), \bar{h}\left(F_{q}(D, \bar{R})\right)=F_{q}\left(D^{\prime}, \bar{R}^{\prime}\right)$ where $\bar{h}\left(x_{0}, \ldots, x_{b-1}\right):=\left(h\left(x_{0}\right)\right.$, $\left.\ldots, h\left(x_{b-1}\right)\right)$.

As we pointed out in the introduction, an important consequence of this definition is that relational query languages cannot count. For instance, the query that computes the bill of materials (Figure 2) does not belong to a relational query language because its range is infinite. Below, a fixpoint query language is given as an example of such a relational query language. There are other fixpoint query languages which all have the same expressive power [18], [20].

The fixpoint query language $F O+L F P$ encompasses a fixpoint operator. Let $(D, \bar{R})$ be an instance of a relational database as described above. Furthermore, let $\varphi(P, \bar{x})$ be a first-order formula with a distinguished $r$-ary predicate symbol $P$ and $r$ free variables $\bar{x}=$ 
$\left(x_{0}, \ldots, x_{r-1}\right)$. In addition to $P$ and $\bar{x}$, the formula $\varphi(P, \bar{x})$ may also contain $\wedge, \vee, \neg, \exists, \forall, R_{0} \ldots, R_{k-1}$, and bound variables. The formula $\varphi(P, \bar{x})$ is assumed to contain only positive occurrences of $P$. Thus, the operator $\tau$ given by $\tau(P):=\left\{\bar{d} \in D^{r} \mid \varphi(P, \bar{d})\right\}$ has a least fixpoint $\varphi_{\infty}:=l f p(\tau)$ because $\tau$ is monotonic and $D$ is finite. When the first-order query language $F O$ is augmented by a fixpoint operator, the fixpoint query language $F O+L F P$ is obtained which is much more expressive than the first-order query language $F O$ (Figure 1). The fixpoint operator determines the semantics of $P$ occurring in a first-order formula $\varphi(P, \bar{x})$. The fixpoint query language $F O+L F P$ can be restricted to formulas containing the fixpoint operator at most once [20].

In what follows, two examples of fixpoint queries are given. First, when the fixpoint operator is applied to

$$
\varphi\left(P,\left(x_{0}, x_{1}\right)\right):=R\left(x_{0}, x_{1}\right) \vee(\exists x)\left(R\left(x_{0}, x\right) \wedge P\left(x, x_{1}\right)\right),
$$

the transitive closure of $R$ is obtained (query TC in Figure 1). Second, applying the fixpoint operator to

$$
\begin{aligned}
& \psi\left(P,\left(x_{0}\right)\right) \\
& \quad:=B\left(x_{0}\right) \vee(\exists x)\left(E\left(x_{0}, x\right) \wedge\left(\forall x^{\prime}\right)\left(E\left(x, x^{\prime}\right) \rightarrow P\left(x^{\prime}\right)\right)\right)
\end{aligned}
$$

yields a least fixpoint which determines whether player I has a winning strategy or not. The relation $E$ represents the edges of the tree and $B$ represents the black leave nodes. Let $\psi_{\infty}$ be the least fixpoint and $r$ be the root of the tree. Then, $\psi_{\infty}(r)$ is the query Game in Figure 1.

The query Game cannot be formulated in Datalog with stratified negation [10]. However, if the body of the rules may contain quantifiers [29], the query Game can be expressed by means of the following rules.

$$
\begin{aligned}
& \operatorname{win}(S) \leftarrow \operatorname{black}(S) . \\
& \operatorname{win}(S) \leftarrow \operatorname{move}(S, T) \wedge \forall U:(\operatorname{move}(T, U) \rightarrow \operatorname{win}(U)) .
\end{aligned}
$$

Let $Q L_{1}$ and $Q L_{2}$ be two relational query languages which belong to the information systems $I S_{1}=\left\langle D B S_{r e l}, Q L_{1}, A S_{r e l}, \alpha_{1}\right\rangle$ and $I S_{2}=\left\langle D B S_{\text {rel }}, Q L_{2}, A S_{r e l}, \alpha_{2}\right\rangle$ respectively. According to Chandra and Harel, $Q L_{2}$ is as expressive as $Q L_{1}$ iff $\left\{F_{q_{1}} \mid q_{1} \in Q L_{1}\right\} \subseteq\left\{F_{q_{2}} \mid\right.$ $\left.q_{2} \in Q L_{2}\right\}$. The following proposition shows that this notion of expressive power is equivalent to our notion of expressive power. 
Proposition 5 Let $I S_{1}=\left\langle D B S, Q L_{1}, A S, \alpha_{1}\right\rangle$ and $I S_{2}=\langle D B S$, $\left.Q L_{2}, A S, \alpha_{2}\right\rangle$ be two information systems that are based on the same database system and on the same answer system. Furthermore, let $I_{1}: D B S \rightarrow D B S$ and $I_{2}: A S \rightarrow A S$ be the identity mappings, i.e. $I_{1}(d b)=d b$ and $I_{2}(a)=a$. Then, $Q L_{1}\left(I S_{1}\right) \leq_{I_{1}, I_{2}} Q L_{2}\left(I S_{2}\right)$ iff $\mathcal{F}_{1}:=\left\{F_{q_{1}}: D B S \rightarrow A S, d b \mapsto \alpha_{1}\left(d b, q_{1}\right) \mid q_{1} \in Q L_{1}\right\} \subseteq \mathcal{F}_{2}:=\left\{F_{q_{2}}:\right.$ $\left.D B S \rightarrow A S, d b \mapsto \alpha_{2}\left(d b, q_{2}\right) \mid q_{2} \in Q L_{2}\right\}$.

Proof. From $Q L_{1}\left(I S_{1}\right) \leq I_{I_{1}, I_{2}} Q L_{2}\left(I S_{2}\right)$ follows $F_{q_{1}}=F_{h\left(q_{1}\right)}$ and hence, $\mathcal{F}_{1} \subseteq \mathcal{F}_{2}$. Conversely, from $\mathcal{F}_{1} \subseteq \mathcal{F}_{2}$ follows that for every $q_{1} \in$ $Q L_{1}$ there exists a $q_{2} \in Q L_{2}$ such that $F_{q_{1}}=F_{q_{2}}$. Hence, there exists a mapping $h: Q L_{1} \rightarrow Q L_{2}$ such that $F_{q_{1}}=F_{h\left(q_{1}\right)}$ for all $q_{1} \in Q L_{1}$. The existence of such a mapping implies that $Q L_{1}\left(I S_{1}\right) \leq_{I_{1}, I_{2}} Q L_{2}\left(I S_{2}\right)$. $\diamond$

Using the proposition above, the results of various authors on the expressive power of relational query languages can be summarized in the following way (see also Figure 1).

$$
\begin{array}{rll}
F O & =_{I_{1}, I_{2}} & \text { RelAlg } \\
F O & <_{I_{1}, I_{2}} & \text { Datalog } \\
\text { Datalog } & <_{I_{1}, I_{2}} & \text { Datalog } \\
\text { Datalog } & <_{I_{1}, I_{2}} & \text { FO+LFP } \\
F O+L F P & <_{I_{1}, I_{2}} & \text { QPTIME }
\end{array}
$$

First-order queries $\varphi \in F O$ are first-order formulas. The answer to a first-order query $\varphi$ is $\{\bar{d} \mid \varphi(\bar{d})\}$. As shown in [12], FO and relational algebra, RelAlg, are equally expressive. If $\langle\rangle,, \leq$, and $\geq$ are excluded, $F O$ is less expressive than Datalog $\urcorner$, i.e. Datalog with stratified negation [3]. This fact is shown in [1]. Whether or not the transitive closure can be expressed in RelAlg or in $F O$ when $\langle$,$\rangle ,$ $\leq$, and $\geq$ are available seems to be an open question (see also comment in [17]). Negation is not allowed in pure Datalog [3]. Due to the missing negation, the query NotTC is not expressible in Datalog (Figure 1). Thus, Datalog $\urcorner$ is more expressive than pure Datalog. Furthermore, the fixpoint query language $F O+L F P$ is more expressive than Datalog [21]. Finally, $F O+L F P$ is less expressive than QPTIME [10]. 


\section{Weighted Query Languages}

In this section, we compare the expressive power of a Boolean query language with the expressive power of a weighted query language where every query consists of vague search criteria. We will present a necessary and sufficient condition which characterizes the cases where the Boolean query language is less expressive than the weighted query language.

The Boolean query language and the weighted query language are of different types. The answer to a Boolean query is a set of data items, i.e. the set of items that satisfy a given Boolean condition: On the other hand, the answer to a weighted query is a function that assigns every data item a numerical value. This numerical value expresses the degree up to which the specified search criteria are satisfied.

Let $D=\left\{d_{0}, \ldots, d_{n-1}\right\}$ be the set of stored data items and let $\Phi=$ $\left\{\varphi_{0}, \ldots, \varphi_{m-1}\right\}$ be a set of search criteria. Every search criterion $\varphi_{i}$ is a function $\varphi_{i}: D \rightarrow[0,1], d_{j} \mapsto \varphi_{i}\left(d_{j}\right)$. The value $\varphi_{i}\left(d_{j}\right)$ expresses how well the search criterion $\varphi_{i}$ is satisfied by $d_{j}$. For instance, let $\varphi_{i}$ be the search criterion age $\left(d_{j}\right)=20$. The function $\varphi_{i}$ can be modeled by means of a regression function which yields $\varphi_{i}\left(d_{j}\right)=1.0$ if age $\left(d_{j}\right)=20$. The value $\varphi_{i}\left(d_{j}\right)$ is decreasing when $\left|a g e\left(d_{j}\right)-20\right|$ is increasing [15].

For the subsequent analysis we have to know only how well the search criteria are satisfied by the data items. Hence, every data item $d_{j}$ can be represented as a vector

$$
d_{j}=\left(\varphi_{0}\left(d_{j}\right), \ldots, \varphi_{m-1}\left(d_{j}\right)\right)
$$

We assume that the data items are uniquely identified by these vectors such that every instance of the database system can be represented by an $m \times n$ matrix $A$ where

$$
A_{i, j}:=\varphi_{i}\left(d_{j}\right)
$$

According to our assumption, the data item is uniquely iclentified by the the $j^{\text {th }}$ column of the matrix $A$. The database system $\mathcal{A}$ contains some matrices $A$, each representing an instance of the database system.

A Boolean query is a Boolean expression of search criteria. Thus, every search criterion $\varphi_{i}$ is a Boolean query and $\left(q \wedge q^{\prime}\right),\left(q \vee q^{\prime}\right)$, and $(\neg q)$ are Boolean queries if $q$ and $q^{\prime}$ are Boolean queries. The set of all Boolean queries is denoted by $Q L_{b}$. 
The answer to a Boolean query consists of a set of data items. The power set of $D$ is denoted by $A S_{b}$ which represents the answer system. Thus, $A S_{b}$ contains all possible answers to Boolean queries.

The Boolean query evaluation function $\alpha_{b}: \mathcal{A} \times Q L_{b} \rightarrow A S_{b}$ is defined inductively.

$$
\begin{aligned}
\alpha_{b}\left(A, \varphi_{i}\right) & \left.:=\left\{d_{j} \in D \mid A_{i, j}=1\right\}\right) \\
\alpha_{b}\left(A, q \wedge q^{\prime}\right) & :=\alpha_{b}(A, q) \cap \alpha_{b}\left(A, q^{\prime}\right) \\
\alpha_{b}\left(A, q \vee q^{\prime}\right) & :=\alpha_{b}(A, q) \cup \alpha_{b}\left(A, q^{\prime}\right) \\
\alpha_{b}(A, \neg q) & :=D-\alpha_{b}(A, q)
\end{aligned}
$$

The Boolean information system is represented by the following structure.

$$
B I S=\left\langle\mathcal{A}, Q L_{b}, A S_{b}, \alpha_{b}\right\rangle
$$

As an example of a Boolean information system, we may think of a Boolean full-text retrieval system. Every search criterion $\varphi_{i}$ corresponds to a keyword which either occurs in the document $d_{j}$ or not. In the former case, $\varphi_{i}\left(d_{j}\right)=1$ and in the latter case $\varphi_{i}\left(d_{j}\right)=0$.

A weighted query is a vector $q=\left(q_{0}, \ldots, q_{m-1}\right)^{T}$ where each component $q_{i}$ is a weight expressing the importance of the search criterion $\varphi_{i}$. The weighted query language $Q L_{w}$ consists of all $m$-dimensional vectors, i.e. $Q L_{w}=\mathrm{R}^{m}$.

The answer to a weighted query consists of a vector of so-called retrieval status values.

$$
\alpha_{w}(A, q):=\left(R S V_{w}\left(q, d_{0}\right), \ldots, R S V_{w}\left(q, d_{n-1}\right)\right)
$$

The retrieval status value $R S V_{w}\left(q, d_{j}\right)$ expresses how well the search criteria specified by the query $q$ are satisfied by the data item $d_{j}$. Such an answer can also be considered as a fuzzy set which specifies for every data item the probability that the item belongs to the answer set. As shown in [24], meaningful retrieval status values can be obtained by means of an appropriate positive definite $m \times m$ matrix $M$.

$$
R S V_{w}\left(q, d_{j}\right):=\sum_{h, i} q_{h} M_{h, i} A_{i, j}
$$

In matrix notation, equation (33) becomes $\alpha_{w}(A, q)=q^{T} M A$. In the simplest case, $M$ is the identity matrix and the retrieval status value 
is equal to the inner vector product of the query vector $q$ and the item vector $d_{j}$. In this case, we have

$$
R S V_{w}\left(q, d_{j}\right):=q_{0} A_{0, j}+\ldots+q_{m-1} A_{m-1, j}
$$

and equation (33) becomes $\alpha_{w}(A, q)=q^{T} A$. Equation 35 shows that the retrieval status value is maximal if all specified search criteria are satisfied. The objective of weighted retrieval is to achieve that the more the user knows about the desired data items the more likely he or she will find them. Unfortunately, Boolean information systems do not always have this behavior: The more the user knows about the desired data items and the more search criteria the user specifies, the more likely it happens that one of the specified search criteria is not completely correct. In this case, the desired data items are not retrieved by a Boolean information system. This problem is usually circumvented by artificial keys (e.g. employee numbers or social security numbers) such that the set of the desired data'items can be specified by very few search criteria.

Let $A S_{w}$ denote the set of possible answers to weighted queries. Thus, $A S_{w}$ contains vectors consisting of $n$ retrieval status values. We specify a weighted retrieval system in the following way.

$$
W I S=\left\langle\mathcal{A}, Q L_{w}, A S_{w}, \alpha_{w}\right\rangle
$$

Our main result on the expressive power of Boolean retrieval and weighted retrieval will be based on the proposition given below. This proposition contains a necessary and sufficient condition which characterizes the cases where every Boolean query can be replaced by a weighted query. The proof of the proposition is given in Appendix A.

Proposition 6 Let $q_{b}$ be any Boolean query and let $J_{q}$ be the set of indices of those items $d_{j}$ that satisfy $q_{b}$, i.e. $J_{q}:=\left\{j \mid d_{j} \in \alpha_{b}\left(A, q_{b}\right)\right\}$. There exists a weighted query $q_{w}$ such that for all data items $d_{j}$ in $D$,

$$
R S V_{w}\left(q_{w}, d_{j}\right)= \begin{cases}1.0 & \text { if } d_{j} \in \alpha_{b}\left(A, q_{b}\right) \\ 0.0 & \text { otherwise }\end{cases}
$$

if and only if

$$
\begin{gathered}
j \in J_{q} \wedge d_{j}=\sum_{h \in J_{q}-\{j\}} c_{h} d_{h} \Longrightarrow \sum_{h \in J_{q}-\{j\}} c_{h}=1, \\
j \notin J_{q} \wedge d_{j}=\sum_{h=0}^{n-1} c_{h} d_{h} \Longrightarrow \sum_{h \in J_{q}} c_{h}=0 .
\end{gathered}
$$


The Boolean information system $B I S=\left\langle\mathcal{A}, Q L_{b}, A S_{b}, \alpha_{b}\right\rangle$ and the weighted information system $W I S=\left\langle\mathcal{A}, Q L_{w}, A S_{w}, \alpha_{w}\right\rangle$ are based on the same database system $\mathcal{A}$; however, their answer systems are of different types. Let $A$ be a non-zero matrix representing a nontrivial instance of the database system. The Boolean queries generate a finite set of answers, i.e. $\left\{\alpha_{b}\left(A, q_{b}\right) \mid q_{b} \in Q L_{b}\right\}$ is finite; however, the weighted queries generate an uncountable infinite set of answers, i.e. $\left\{\alpha_{w}\left(A, q_{w}\right) \mid q_{w} \in Q L_{w}\right\}$ is uncountably infinite.

In what follows, we compare the expressive power of $Q L_{b}$ and the expressive power of $Q L_{w}$. We define the correspondences between the answers of $A S_{b}$ and the answers of $A S_{w}$ as follows.

$$
g: A S_{b} \rightarrow A S_{w}, D^{\prime} \mapsto\left(\chi_{D^{\prime}}\left(d_{0}\right), \ldots, \chi_{D^{\prime}}\left(d_{n-1}\right)\right)
$$

The characteristic function $\chi_{D^{\prime}}: D \rightarrow \mathrm{R}$ of the subset $D^{\prime} \subseteq D$ is defined as usual.

$$
\chi_{D^{\prime}}\left(d_{j}\right)= \begin{cases}1 & \text { if } d_{j} \in D^{\prime} \\ 0 & \text { otherwise }\end{cases}
$$

The correspondences between the instances of the database are given by the identity mapping $I_{1}: \mathcal{A} \rightarrow \mathcal{A}, A \mapsto A$.

Proposition 7 Let $B I S=\left\langle\mathcal{A}, Q L_{b}, A S_{b}, \alpha_{b}\right\rangle$ be a Boolean Information System such that range $\left(\alpha_{b}\right)$ contains more than one element and let $W I S=\left\langle\mathcal{A}, Q L_{w}, A S_{w}, \alpha_{w}\right\rangle$ be a Weighted Information System. Furthermore, let $I_{1}: \mathcal{A} \rightarrow \mathcal{A}$ and $g: A S_{b} \rightarrow A S_{w}$ be the functions defined above. These functions determine the correspondences between the instances of the databases and the correspondences between the answers. The Weighted Query Language $Q L_{w}$ of WIS is more expressive than the Boolean Query Language $Q L_{b}$ of BIS with respect the correspondences given by $I_{1}$ and $g$ iff all instances $A$ of the database system $\mathcal{A}$ satisfy the conditions (38) and (39).

Proof. Assume that all $A \in \mathcal{A}$ satisfy the conditions (38) and (39). $Q L_{b}(B I S) \leq_{I_{1}, g} Q L_{w}(W I S)$ follows immediately from Proposition 6 . Because range $\left(\alpha_{b}\right)$ contains more than one element, there exists a query $q_{w}$ and a document $d_{j}$ such that $R S V_{w}\left(q_{w}, d_{j}\right) \neq 0$. Hence, $q_{w}$ or $q_{w}+q_{\dot{w}}$ do not have a corresponding query in $Q L_{b}$ because either $0<R S V_{w}\left(q_{w}, d_{j}\right) \neq 1$ or $0<R S V_{w}\left(q_{w}+q_{w}, d_{j}\right) \neq 1$. From (12) follows that $Q L_{b}$ and $Q L_{w}$ are not equally expressive. 
Assume that $Q L_{b}(B I S)<I_{1}, g Q L_{w}(W I S)$. By definition, for every Boolean query $q_{b}$ there is a corresponding weighted query $q_{w}$ such that $\forall A \in \mathcal{A}: g\left(\alpha_{b}\left(A, q_{b}\right)\right)=\alpha_{w}\left(A, q_{w}\right)$. From Proposition 6 follows that $(38)$ and (39) are satisfied. $\diamond$

The propositions presented in this section show that there are redundancies between Boolean query languages and weighted query languages. A necessary and sufficient condition was given which determines the cases where a Boolean query can always be replaced by an equivalent weighted query. In DB-theory, redundant data are related to inconsistent data [26, pp. 211]. From an algebraic point of view, the question arises whether redundancies between query languages are related to inconsistencies between query languages. Indeed, there are inconsistencies in the case of Boolean retrieval and weighted retrieval . The problem of these inconsistencies is the separability problem [8] which has been known in Information Retrieval for many years. Problems of the same type also occur in probabilistic databases [5] and in databases with maybe tuples [14]. Analogously to database design theory, we may argue that the separability problem would not occur if there were no redundancies between Boolean retrieval and weighted retrieval.

\section{The Query Language FQL*}

In this section, we introduce the Functional Query language $\mathrm{FQL}^{*}$ as an example of a non-relational query language that provides a simple form of counting. Thus, as pointed out in Section 1, Chandra and Harel's notion of expressive power cannot be used to compare the expressive power of $\mathrm{FQL}^{*}$ with the expressive power of relational query languages. In Section 6, we use our notion of expressive power to show that $\mathrm{FQL}$ is more expressive than the fixpoint query languages.

We do not describe the full language $F Q L^{*}$. We restrict ourselves to those parts of $\mathrm{FQL}^{*}$ which determine its expressive power. The syntax of FQL* is similar to the syntax of OSQL [6], [28]. Unlike the specifications of OSQL functions, the specifications of $F Q L^{*}$ functions can be recursive or even mutually recursive. Like Datalog, $\mathrm{FQL}^{*}$ has a fixpoint semantics that can be computed within finite time or more precisely, within a time bounded by a polynomial function of the size of the database. In this section, we give examples that show how the ancestor function and the bill of materials are formulated in FQL*. 


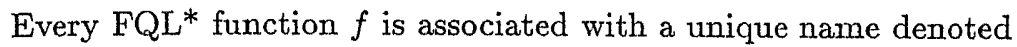
by name $(f)$. There are three different types of FQL* functions: stored functions, built-in functions, and derived functions. Similarly to OSQL [6], stored functions are stored by means of tables. For instance, a table may store the parents of persons. A built-in function is defined procedurally. For instance, given some numbers, the built-in function sum computes the sum of these numbers. Finally, derived functions are defined in a declarative way as described below.

$\mathrm{FQL}^{*}$ functions are bag functions defined as follows. A bag $A$ is a multiset which may contain an element $y$ of a domain $Y$ more than once [2]. In our case, a bag may even contain an element infinitely many times. The number of occurrences of an element $y$ in a bag $A$ is denoted by $A(y)$. Let $\bar{N}:=\{0,1,2, \ldots, \omega\}$ be the set of natural numbers together with the limit ordinal $\omega$ denoting infinity. Every bag $A$ is represented as a function $A: Y \rightarrow \bar{N}, y \mapsto A(y)$. The set of all bags with domain $Y$ is denoted by $B(Y)$. A bag $A \in B(Y)$ is called a finite bag iff $\sum_{y \in Y} A(y)<\omega$. Subsequently, finite bags are written like sets, except that an element may occur several times (e.g. $A=\{a, a, b\})$. A bag $A \in B(Y)$ is contained in a bag $A^{\prime} \in B(Y)$ iff every element $y \in Y$ occurs in $A^{\prime}$ at least as many times as in $A$.

$$
A \sqsubseteq A^{\prime} \quad: \Longleftrightarrow \forall y: A(y) \leq A^{\prime}(y)
$$

Given a natural number $n$ and two bags $A, A^{\prime} \in B(Y)$, the intersection $A \sqcap A^{\prime}$, the union $A \sqcup A^{\prime}$, the sum $A+A^{\prime}$, and the multiple $n * A$ are defined as follows.

$$
\begin{gathered}
A \sqcap A^{\prime}: Y \rightarrow \bar{N}, y \mapsto \min \left(A(y), A^{\prime}(y)\right) \\
A \sqcup A^{\prime}: Y \rightarrow \bar{N}, y \mapsto \max \left(A(y), A^{\prime}(y)\right) \\
A+A^{\prime}: Y \rightarrow \bar{N}, y \mapsto A(y)+A^{\prime}(y) \\
n * A: Y \rightarrow \bar{N}, y \mapsto n * A(y)
\end{gathered}
$$

A bag function is a functional whose range is a set of bags. We will restrict ourselves to bag functions of the following types

$$
\begin{gathered}
f: X \rightarrow B(Y) \\
g: B(X) \rightarrow B(Y)
\end{gathered}
$$

where $X$ and $Y$ are sets of objects or, in the terminology of objectoriented database systems, $X$ and $Y$ are classes. Every function value $f(x)$ is a bag, i.e. a mapping from $Y$ to $\bar{N}$, and $f(x)(y)$ is the number 


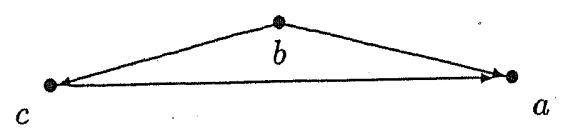

Figure 5: A directed graph.

of occurrences of $y$ in the bag $f(x)$. The following example shows two bag functions associated with a directed graph.

Example 3 Let $G=\langle V, E\rangle$ be the graph with vertices $V$ and edges $E \subseteq V \times V$ as shown in Figure 5. The successor function $s: V \rightarrow$ $B(\bar{V}), v \mapsto s(v)$ determines the bag of immediate successor vertices for every vertex.

$$
s(a)=\{\} \quad s(b)=\{a, c\} \quad s(c)=\{a\}
$$

The bag function $p: V \rightarrow B(V), v \mapsto p(v)$ assigns every vertex $v$ a bag $p(v)$ which contains a vertex $v^{\prime}$ as many times as there are different paths from $v$ to $v^{\prime}$.

$$
p(a)=\{\} \quad p(b)=\{a, a, c\}, p(c)=\{a\}
$$

If the graph $G$ represents a "part-of" relation, $s(v)$ contains the immediate subparts of $v$ and $p(v)$ contains all subparts of $v$, i.e. $p(v)\left(v^{\prime}\right)$ determines how many times the part $v^{\prime}$ is contained in part $v$.

Given a natural number $n$ and two bag functions $f: X \rightarrow B(Y)$ and $g: X \rightarrow B(Y)$, the intersection $f \sqcap g$, the union $f \sqcup g$, the sum $f+g$, and the multiple $n * f$ are defined as follows.

$$
\begin{gathered}
f \sqcap g: X \rightarrow B(Y), x \mapsto f(x) \sqcap g(x) \\
f \sqcup g: X \rightarrow B(Y), x \mapsto f(x) \sqcup g(x) \\
f+g: X \rightarrow B(Y), x \mapsto f(x)+g(x) \\
n * f: X \rightarrow B(Y), x \mapsto n * f(x)
\end{gathered}
$$

Given a set of bag functions mapping $X$ to $B(Y)$, we define a partial ordering $\sqsubseteq$ on this set. Let $f: X \rightarrow B(Y)$ and $g: X \rightarrow B(Y)$ be such bag functions. Using (42), the relation $\sqsubseteq$ on bag functions is defined by means of the relation $\sqsubseteq$ on bags.

$$
f \sqsubseteq g \quad: \Longleftrightarrow \forall x \in X: f(x) \sqsubseteq g(x)
$$




\begin{tabular}{|c|c||c|c||c|c|}
\hline \multicolumn{2}{|c||}{ Table $F$} & \multicolumn{2}{c||}{ Table $H$} & \multicolumn{2}{c|}{ Table $F H$} \\
\hline$X$ & $Y$ & $Y$ & $Z$ & $X$ & $Z$ \\
\hline$a$ & $p$ & $p$ & $u$ & $a$ & $u$ \\
$b$ & $p$ & $p$ & $v$ & $a$ & $v$ \\
$b$ & $q$ & $q$ & $v$ & $b$ & $u$ \\
& & $\vdots$ & $\vdots$ & $b$ & $v$ \\
& & $q$ & $v$ & $\vdots$ & $\vdots$ \\
& & & & $b$ & $v$ \\
\hline
\end{tabular}

Figure 6: The tables $F, H$, and $F H$ represent the bag functions $f$ : $X \rightarrow B(Y), x \mapsto f(x), h: Y \rightarrow B(Z), y \mapsto h(y)$, and $h \diamond f: X \rightarrow$ $B(Z), x \mapsto h(f(x))$ respectively.

FQL* uses a generalized form of composition of bag functions. The generalized form facilitates not only the composition of functions $f: X \rightarrow B(Y)$ and $g: B(Y) \rightarrow B(Z)$, but also the composition of functions $f: X \rightarrow B(Y)$ and $h: Y \rightarrow B(Z)$. Before defining $h(f(x))$ in terms of bags, we define it by means of relational algebra. Assume that the bag functions $f$ and $h$ are represented conceptually by the tables $F$ and $H$ (Figure 6). These are conceptual representations because, for instance, the table $H$ contains the tuple $(q, v)$ infinitely many times. See the proof of Proposition 9 for the tables that are actually used to compute the composition.

The tables $F$ and $H$ determine the bag functions $f$ and $h$ by

$$
\begin{aligned}
& f: X \rightarrow B(Y), x \mapsto \Pi[Y](\sigma[X=x](F)) \\
& h: Y \rightarrow B(Z), y \mapsto \Pi[Z](\sigma[Y=y](H))
\end{aligned}
$$

where $\sigma$ is the conventional select operator and $\Pi$ is a special project operator which does not remove duplicates. In this way, the bag function $h$ assigns the element $q$ the bag $h(q)$ which contains $v$ infinitely many times, i.e. $h(q)(v)=\omega$. The composition $h(f(x))$ is based on the natural join of $F$ and $H$ (the join attribute is $Y$ ).

$$
h(f(x)):=\Pi[Z](\sigma[X=x](F \bowtie H))
$$

The resulting function $h \diamond f: X \rightarrow B(Z), x \mapsto h(f(x))$ is represented by the table $F H$ (Figure 6 ).

In what follows, we define the generalized composition in terms of bags. Let $f: X \rightarrow B(Y), g: B(Y) \rightarrow B(Z)$, and $h: Y \rightarrow B(Z)$ be 
three bag functions. We define the compositions $g \circ f$ and $h \diamond f$ as follows.

$$
\begin{gathered}
g \circ f: X \rightarrow B(Z), x \mapsto g(f(x)) \\
h \diamond f: X \rightarrow B(Z), x \mapsto \sum_{y} f(x)(y) * h(y)
\end{gathered}
$$

Note that the function value $\sum_{y} f(x)(y) * h(y)$ is defined by (46) and (45). The bag $h \diamond f(x)$ is obtained by determining the bag $f(x)$ and then adding up the bags $h(y)$ for every occurrence of $y$ in $f(x)$. Sometimes, the composition $h \diamond f$ is called flattening because the bag $f(x)$ is made flat and $h$ is applied to every occurrence of an element $y$ in $f(x)$. For instance, assume that $f(a)=\{b, b, c\}$. Then, $h$ is applied to every occurrence of an element in $f(a)$ to obtain, say $h(b)=\{d, d\}$ for the first occurrence of $b$ in $f(a), h(b)=\{d, d\}$ for the second occurrence of $b$ in $f(a)$, and $h(c)=\{d, e\}$ for the occurrence of $c$ in $f(a)$. Adding up the $h$ values yields $(h \diamond f)(a)=h(b)+h(b)+h(c)=\{d, d, d, d, d, e\}$. We will use the following notational convention.

$$
f^{\prime \prime}\left(f^{\prime}(x)\right):= \begin{cases}f^{\prime \prime} \circ f^{\prime}(x) & \text { if } \operatorname{range}\left(f^{\prime}\right) \subseteq \operatorname{dom}\left(f^{\prime \prime}\right) \\ f^{\prime \prime} \diamond f^{\prime}(x) & \text { if } \operatorname{range}\left(f^{\prime}\right) \subseteq B\left(\operatorname{dom}\left(f^{\prime \prime}\right)\right)\end{cases}
$$

Example 4 Let $A=\{1,1,8,9\}$ be a bag of $B$ (int) and let inc and sum be two bag functions defined as follows.

$$
\begin{aligned}
\text { inc }: \text { int } \rightarrow B(\text { int }), i & \mapsto\{i+1\} \\
\text { sum }: B(\text { int }) \rightarrow B(\text { int }), X & \mapsto\left\{\sum_{i} X(i) * i\right\}
\end{aligned}
$$

According to the generalized composition (59), we obtain, for instance, $\operatorname{inc}(7)=\{8\}, \operatorname{inc}(A)=\{2,2,9,10\}, \operatorname{sum}(A)=\{19\}, \operatorname{sum}(\operatorname{inc}(A))=$ $\{23\}$, and $\operatorname{inc}(\operatorname{sum}(A))=\{20\}$.

We use a slightly modified version of the OSQL syntax [6] to specify derived $\mathrm{FQL}^{*}$ functions. A set of $n$ derived $\mathrm{FQL}^{*}$ functions $f_{0}, \ldots, f_{n-1}$ is specified by $n$ create statements each of the following form where $0 \leq q_{i} \leq r_{i} \leq s_{i}$.

create derived function

$f_{i}: X(i, 0) \times \ldots \times X\left(i, q_{i}-1\right) \rightarrow B\left(Y(i, 0) \times \ldots \times Y\left(i, p_{i}-1\right)\right)$

such that $f_{i}\left(x_{0}, \ldots, x_{q_{i}-1}\right)$ entails $\chi_{i}\left(x_{q_{i}}, \ldots, x_{r_{i}-1}\right)$

for each $\left(x_{q_{i}}, \ldots, x_{r_{i}}, \ldots, x_{s_{i}-1}\right)$ in $X\left(i, q_{i}\right) \times \ldots \times X\left(i, s_{i}-1\right)$

where $\varphi_{i}(\bar{f})\left(x_{0}, \ldots, x_{q_{i}-1}, x_{q_{i}}, \ldots, x_{r_{i}-1}, x_{r_{i}}, \ldots, x_{s_{i}-1}\right)$; 
The sets $X(i, j)$ and $Y(i, j)$ denote sets of objects. The keyword "entails" denotes the relation $\sqsupseteq$ and $\chi_{i}$ denotes a composition of functions which maps $X\left(i, \underline{q}_{i}\right) \times \ldots \times X\left(i, r_{i}-1\right)$ to $B\left(Y(i, 0) \times \ldots \times Y\left(i, p_{i}-\right.\right.$ $1))$. The predicate $\varphi_{i}(\bar{f}): X(i, 0) \times \ldots \times X\left(i, s_{i}-1\right) \rightarrow\{$ false, true $\}$ may depend on the functions $\bar{f}=\left(f_{0}, \ldots, f_{n-1}\right)$. This predicate is a propositional expression (and, or, not) of atomic formulas of the form $\left(\chi(\bar{z})\right.$ entails $\left.\chi^{\prime}\left(\bar{z}^{\prime}\right)\right)$, where $\chi$ and $\chi^{\prime}$ are compositions of functions. We will use the following abbreviations.

$$
\begin{aligned}
\left(\chi(\bar{z})=\chi^{\prime}\left(\bar{z}^{\prime}\right)\right):= & \left(\chi(\bar{z}) \text { entails } \chi^{\prime}\left(\bar{z}^{\prime}\right)\right) \\
& \text { and }\left(\chi^{\prime}\left(\bar{z}^{\prime}\right) \text { entails } \chi(\bar{z})\right) \\
\left(\bar{z}=\bar{z}^{\prime}\right):= & \left(\{\bar{z}\}=\left\{\bar{z}^{\prime}\right\}\right) \\
\left(\chi(\bar{z}) \text { contains } \bar{z}^{\prime}\right):= & \left(\chi(\bar{z}) \text { entails }\left\{\bar{z}^{\prime}\right\}\right)
\end{aligned}
$$

Definition 6 The predicate $\varphi_{i}$ is called monotonic iff

$$
\bar{f} \sqsubseteq \bar{f}^{\prime} \Longrightarrow\left(\varphi_{i}(\bar{f}) \Rightarrow \varphi_{i}\left(\bar{f}^{\prime}\right)\right)
$$

where $\bar{f} \sqsubseteq \bar{f}^{\prime}$ denotes that $f_{i} \sqsubseteq f_{i}^{\prime}$ for $0 \leq i<n$. A bag function $g$ mapping either $X$ to $B(Y)$ or $B(X)$ to $B(Y)$ is called monotonic iff $A \sqsubseteq A^{\prime}$ implies that $g(A) \sqsubseteq g\left(A^{\prime}\right)$ for all bags $A$ and $A^{\prime}$ in $B(X)$.

Proposition 8 Every bag function $f: X \rightarrow B(Y)$ is monotonic.

Proof. $A \sqsubseteq A^{\prime} \Rightarrow \forall x: A(x) \leq A^{\prime}(x) \Rightarrow \forall x: \forall y: A(x) * f(x)(y) \leq$ $A^{\prime}(x) * f(\bar{x})(y) \Rightarrow \forall y: f(A)(y)=\sum_{x} A(x) * f(x)(y) \leq \sum_{x} A^{\prime}(x) *$ $f(x)(y)=f\left(A^{\prime}\right)(y) \Rightarrow f(A) \sqsubseteq f\left(A^{\prime}\right) . \diamond$

From the proposition above follows that a non-monotonic function is of the type $g: B(X) \rightarrow B(Y)$. For instance, the function sum $: B($ int $) \rightarrow B($ int $)$ introduced in Example 4 is not monotonic because $\{1,2\} \sqsubseteq\{1,2,3\}$ but $\operatorname{sum}(\{1,2\})=\{3\} \nsubseteq\{6\}=\operatorname{sum}(\{1,2,3\})$. Before presenting the semantics of the create statements that specify derived functions, we give an example.

Example 5 Assume that the function par: person $\rightarrow B$ (person) is a stored function which determines the parents for every person. The following create statement specifies the ancestor function.

create derived function anc: person $\rightarrow B$ (person)

such that $\operatorname{anc}\left(x_{0}\right)$ contains $x_{1}$

for each $x_{1}$ in person

where $\left(\operatorname{par}\left(x_{0}\right) \sqcup \operatorname{par}\left(\operatorname{anc}\left(x_{0}\right)\right)\right.$ contains $\left.x_{1}\right)$; 
The create statements specifying the derived functions $f_{0}, \ldots, f_{n-1}$ correspond to a system of $n$ inequalities of the form

$$
f_{i}\left(\bar{x}_{i}\right) \sqsupseteq \chi_{i}\left(\pi_{i}\left(\sigma\left(\varphi_{i}(\bar{f}), \bar{x}_{i}\right)\left(X(i, 0) \times \ldots \times X\left(i, s_{i}-1\right)\right)\right)\right) .(61)
$$

where $\bar{x}_{i}=\left(x_{0}, \ldots, x_{q_{i}-1}\right) \in X(i, 0) \times \ldots \times X\left(i, q_{i}-1\right)$ and $\bar{f}=$ $\left(f_{0}, \ldots, f_{n-1}\right)$. The select operator $\sigma\left(\varphi_{i}(\bar{f}), \bar{x}_{i}\right)$ selects from $X(i, 0) \times$ $\ldots \times X\left(i, s_{i}-1\right)$ those tuples $\left(x_{0}^{\prime}, \ldots, x_{s_{i}-1}^{\prime}\right)$ for which $\left(x_{0}^{\prime}, \ldots, x_{q_{i}-1}^{\prime}\right)=$ $\bar{x}_{i}$ and the predicate $\varphi_{i}(\bar{f})\left(x_{0}^{\prime}, \ldots, x_{s_{i}-1}^{\prime}\right)$ is true. The set containing the selected tuples is considered as a bag of $B\left(X(i, 0) \times \ldots \times X\left(i, s_{i}-\right.\right.$ 1)). The function

$$
\pi_{i}: X(i, 0) \times \ldots \times X\left(i, s_{i}-1\right) \rightarrow B\left(X\left(i, q_{i}\right) \times \ldots \times X\left(i, r_{i}-1\right)\right)
$$

"projects" the tuples $\left(x_{0}^{\prime}, \ldots, x_{s_{i}-1}^{\prime}\right)$ to $\left(x_{q_{i}}^{\prime}, \ldots, x_{r_{i}-1}^{\prime}\right)$ without removing duplicates. In contrast to the function $\pi_{i}$, the function $\sigma\left(\varphi_{i}(\bar{f}), \bar{x}_{i}\right)$ cannot generate duplicates. The function

$$
\chi_{i}: X\left(i, q_{i}\right) \times \ldots \times X\left(i, r_{i}-1\right) \rightarrow B\left(Y(i, 0) \times \ldots \times Y\left(i, p_{i}-1\right)\right)
$$

represents a composition of functions. In a special case, the composition $\chi_{i}$ may represent the identity where $\chi_{i}\left(x_{q_{i}}, \ldots, x_{r_{i}-1}\right)$ is equal to $\left\{\left(x_{q_{i}}, \ldots, x_{r_{i}-1}\right)\right\}$. According to Proposition 8 , the functions $\pi_{i}$ and $\chi_{i}$ are monotonic functions. The function $\sigma\left(\varphi_{i}(\bar{f}), \bar{x}_{i}\right)$ is monotonic if the predicate $\varphi_{i}(\bar{f})$ is monotonic.

The inequality (61) reveals a certain similarity with relational algebra which also contains select and project operators. As shown at the outset of this section, the composition of functions corresponds to the natural join. In addition, there exists a similarity between (61) and Datalog. When the predicates $p_{i}$ are considered as relations $P_{i}$, every rule $p_{0} \leftarrow p_{1} \wedge \ldots \wedge p_{k}$ corresponds to an inequality $P_{0} \supseteq P_{1} \cap \ldots \cap P_{k}$.

The create statements specifying the derived functions $f_{0}, \ldots, f_{n-1}$ correspond to $n$ inequalities of the form (61). The semantics of these create statements is given by $n$ bag functions representing a unique minimal solution of the $n$ inequalities. A solution is called a minimal solution iff $\bar{f}^{\prime} \nsubseteq \bar{f}$ for every solution $\bar{f}^{\prime}$ that is different from $\bar{f}$. The following proposition gives a sufficient stratification condition implying the existence of a unique minimal solution. Similarly to Datalog, the uniqueness of the solution is guaranteed by a bottom up computation described in the proof of the proposition which is given in Appendix A. 
Proposition 9 Assume that the functions $f_{0}, \ldots, f_{n-1}$ are specified by $n$ create statements which correspond to a system of $n$ inequalities of the form

$$
f_{i}\left(\bar{x}_{i}\right) \sqsupseteq \chi_{i}\left(\pi_{i}\left(\sigma\left(\varphi_{i}(\bar{f}), \bar{x}_{i}\right)\left(X(i, 0) \times \ldots \times X\left(i, s_{i}-1\right)\right)\right)\right) .
$$

This system of $n$ inequalitics has a uniquely determined minimum solution if there exists a stratification function

$$
\nu:\{0, \ldots, n-1\} \rightarrow\{0, \ldots, n-1\}, i \mapsto \nu(i)
$$

such that for every $i \in\{0, \ldots, n-1\}$, the following three conditions are satisfied.

1. If $f_{j}$ occur's in the specification of $f_{i}$, then $\nu(j) \leq \nu(i)$.

2. The domain $\left.X(i, 0) \times \ldots \times X\left(i, q_{i}-1\right)\right)$ of $f_{i}$ is finite.

3. If $f_{j}$ occurs in the specification of $f_{i}$ and $\nu(j)=\nu(i)$, then $\varphi_{i}$ is monotonic and $Y(i, 0) \times \ldots \times Y\left(i, p_{i}-1\right)$ is finite.

The uniquely determined minimum solution is computable by a polynomial time algorithm, i.e. in time $O(P(|X|))$ where $P$ denotes a polynomial and $|X|$ denotes the number of objects stored in the database.

The example given below contains a derived FQL* function that computes the bill of materials mentioned in the introduction. The bill of materials is the following graph problem. Given a graph, let $P(v)$ be the set of paths $p$ starting at $v=s(p)$ and ending at $t(p)$. The source vertex $s(p)$ and the target vertex $t(p)$ may be identical and the path $p \in P(v)$ may contain cycles. If the cost of a single vertex $v$ are denoted by $\operatorname{cost}(v)$, the total cost of $v$ are defined by

$$
\text { total_cost }(v):=\sum_{p \in P(v)} \operatorname{cost}(t(p)) .
$$

The cost of every vertex is assumed to be non-negative. Thus, the total costs of certain vertices are infinite if the graph contains cycles. Even in the presence of cycles, the "bill of materials" as a graph problem is well defined and the derived functions given below provide the correct answer. According to Proposition 9, these functions are computed within polynomial time even though some function values may consist of infinite bags. Note that, in contrast to our approach, the approach described in [19] does not deal with cycles, i.e. all graphs are assumed to be acyclic. 
Example 6 Assume that subparts : parts $\rightarrow B$ (parts) is a stored function which determines for every part its immediate subparts. The stored function cost : parts $\rightarrow B($ int $)$ determines for every part the non-negative cost of this particular part without its subparts. The derived function all_subparts determines for every part $x$ a bag of parts. This bag contains a part $y$ as many times as $y$ is contained in $x$.

create derived function all_subparts : parts $\rightarrow B($ parts $)$

such that all_subparts $(x)$

entails subparts $(y)+$ subparts(all_subparts $(y))$

for each $y$ in parts

where $(x=y)$;

In the case of the derived function all_subparts, a trivial combination of a selection function and of a "projection" function occurs because

$$
\pi(\sigma(\text { true }, x)(\text { parts } \times \text { parts }))=\{x\}
$$

In such cases, it is convenient to drop "for each ... where ..." to obtain a more compact specification.

create derived function all_subparts : parts $\rightarrow B$ (parts)

such that all_subparts $(x)$

entails subparts $(x)+$ subparts(all_subparts $(x))$;

The derived function total_cost determines, for every part, the cost for the part itself and of all of its subparts.

create derived function total_cost : parts $\rightarrow B($ int $)$

such that total_cost $(x)$

entails $\operatorname{sum}(\operatorname{cost}(\{x\}+$ all_subparts $(x)))$;

We conclude this section by summarizing the main advantages of bag functions. First of all, there exists a fixpoint semantics for derived bag functions. Second, aggregate functions can be represented in a natural way, e.g. $\operatorname{sum}(\{1,1\})=\{2\}$. In relational query languages, more complicated operators (e.g. group by) are required to compute $1+1$ because the bag $\{1,1\}$ is not a relation. Third, bag functions provide a primitive form of counting. Fourth, the availability of infinite bags together with the capability of counting leads to an outstanding expressive power as will be shown in the next section. Finally, every FQL* function is computable in polynomial time. We believe that 
database queries should be evaluable in a reasonable time and therefore, it should not be possible to formulate NP-complete problems such as the traveling salesman problem [16, pp. 211] or non-decidable problems such as Hilbert's tenth problem [13]. As mentioned in [4], a valuable query language is a tradeoff between expressive power and computational complexity.

\section{The Expressive Power FQL*}

In this section, we show that $\mathrm{FQL}^{*}$ is more expressive than the fixpoint query langtnages. The fixpoint query languages have been introduced in Section 3 . We first show that every fixpoint query can be expressed by means of FQL* functions. The correspondence between the predicates $R: D^{a} \rightarrow\{$ false, true $\}$ and FQL* functions $f: D^{a} \rightarrow B(\{1\})$ is defined by

$$
R \simeq f \quad: \Longleftrightarrow \forall \bar{d} \in D^{a}: R(\bar{d}) \Leftrightarrow f(\bar{d}) \neq\{\} .
$$

Let $f: D^{a} \rightarrow B(\{1\})$ be any FQL* function. We define the derived FQL* functions not.f and exists $f$ as follows.

create derived function $\operatorname{not}_{f}: D^{a} \rightarrow B(\{1\})$

such that not $_{f}\left(d_{0}, \ldots, d_{a-1}\right)$ entails $y$

for each $y$ in $\{1\}$

where $f\left(d_{0}, \ldots, d_{a-1}\right)=\{\}$;

create derived function exists $: D^{a-1} \rightarrow B(\{1\})$

such that exists $s_{f}\left(d_{1}, \ldots, d_{a-1}\right)$ entails $y$

for each $\left(d_{0}, y\right)$ in $D \times\{1\}$

where $f\left(d_{0}, \ldots, d_{a-1}\right)$ contains 1 ;

Subsequently, the formulas $R \vee S$ and $\forall x_{0}: R\left(x_{0}, \ldots, x_{a-1}\right)$ are regarded as abbreviations of $\neg(\neg R \wedge \neg S)$ and $\neg\left(\exists x_{0}: \neg R\left(x_{0}, \ldots, x_{a-1}\right)\right)$ respectively. From the following proposition it follows that every firstorder query is expressible in FQL*.

Proposition 10

$$
\begin{aligned}
R \simeq f, S \simeq g & \Longrightarrow R \wedge S \simeq f \sqcap g \\
R \simeq f & \Longrightarrow \neg R \simeq \text { not } f \\
R \simeq f & \Longrightarrow \exists x_{0}: R\left(x_{0}, \ldots, x_{a-1}\right) \simeq \text { exists }_{f}
\end{aligned}
$$


Proof. The first implication is proven as follows.

$$
\begin{aligned}
R \simeq f, S \simeq g & \Longrightarrow R(\bar{d}) \Leftrightarrow f(\bar{d}) \sqsupseteq\{1\}, S(\bar{d}) \Leftrightarrow g(\bar{d}) \sqsupseteq\{1\} \\
& \Longrightarrow R(\bar{d}) \wedge S(\bar{d}) \Leftrightarrow f(\bar{d}) \sqcap g(\bar{d}) \sqsupseteq\{1\} \\
& \Longrightarrow R \wedge S \simeq f \sqcap g
\end{aligned}
$$

The remaining two implications are proven analogously. $\diamond$

Let $\varphi(P, \bar{x})$ be a first-order formula with a distinguished $r$-ary predicate symbol $P$ and $r$ free variables $\bar{x}=\left(x_{0}, \ldots, x_{r-1}\right)$. In addition to $P$ and $\bar{x}$, the formula $\varphi(P, \bar{x})$ may also contain $\wedge, \vee, \neg, \exists, \forall$, $R_{0}, \ldots, R_{k-1}$, and bound variables. The formula $\varphi(P, \bar{x})$ is assumed to contain only positive occurrences of $P$. As shown in Section 3 , the operator $\tau$ given by $\tau(P):=\left\{\bar{d} \in D^{r} \mid \varphi(P, \bar{d})\right\}$ has a least fixpoint $l f p(\tau)$. In what follows, we show that the fixpoint query $l f p(\tau)$ can be expressed by derived $\mathrm{FQL}$ (functions.

Given the relations $\bar{R}=\left(R_{0}, \ldots, R_{k-1}\right)$, let $\bar{g}=\left(g_{0} \ldots, g_{k-1}\right)$ be $k$ stored functions such that $R_{i} \simeq g_{i}$ for $0 \leq i<k$. Furthermore, let $\varphi(P, \bar{x})$ be any first-order formula as described above. According to Proposition 10, there exist derived functions $f_{1}, \ldots, f_{n-1}$ such that

$$
P \simeq f_{0} \Rightarrow\left\{\bar{d} \in D^{r} \mid \varphi(P, \bar{d})\right\} \simeq f_{1} .
$$

The $n-2$ derived functions $f_{2}, \ldots, f_{n-1}$ correspond to subformulas of $\varphi(P, \bar{x})$. The derived function $f_{0}$ specified by

$$
\begin{aligned}
& \text { create derived function } f_{0}: D^{r} \rightarrow B(\{1\}) \\
& \quad \text { such that } f_{0}\left(d_{0}, \ldots, d_{r-1}\right) \text { entails } f_{1}\left(d_{0}, \ldots, d_{r-1}\right) ;
\end{aligned}
$$

corresponds to $l f p(\tau)$, i.e. $l f p(\tau) \simeq f_{0}$, where $f_{0}$ depends on the other derived functions $f_{1}, \ldots, f_{n-1}$.

According to [18], every fixpoint query can be expressed either by a first-order formula or by a formula containing one fixpoint operator and only positive occurrences of the distinguished predicate $P$. Hence, every fixpoint query can be expressed by derived FQL* functions whose specifications satisfy the three conditions of Proposition 9. On the other hand, not every derived $\mathrm{FQL}^{*}$ function can be expressed as a fixpoint query. For instance, the derived function total_cost (Example 6) cannot be expressed by a fixpoint query as shown in the introduction (Figure 2). In the rest of this section, the new notion of expressive power is used to show that $\mathrm{FQL}^{*}$ queries are more expressive than fixpoint queries. 
We start with defining the information system $I S_{F Q L}$ which is accessible through FQL*.

$$
I S_{F Q L}=\left\langle D B S_{F Q L}, Q L_{F Q L}, A S_{F Q L}, \alpha_{F Q L}\right\rangle .
$$

An instance $\bar{g}=\left(g_{0}, \ldots, g_{k-1}\right)$ of the database system $D B S_{F Q L}$, consists of $k$ stored functions. The create statement

create stored function $g: X_{0} \times \ldots \times X_{q-1} \rightarrow B\left(Y_{0} \times \ldots \times Y_{p-1}\right)$;

creates the stored function $g$ which maps every tuple $\left(x_{0}, \ldots, x_{q-1}\right)$ to the empty bag, i.e. for all $\left(x_{0}, \ldots, x_{q-1}\right), g\left(x_{0}, \ldots, x_{q-1}\right)=\{\}$. Nonempty function values are specified by means of insert statements.

insert $\left(y_{0}, \ldots, y_{p-1}\right)$ into $g\left(x_{0}, \ldots, x_{q-1}\right)$;

An FQL* query $\langle S, f n m\rangle$ consists of a set $S$ and of a name $f n m$. The set $S$ contains create statements specifying derived functions and the name $f n m$ denotes a function. The evaluation function $\alpha_{F Q L}$ is defined for the following arguments. Given an instance $\bar{g}=\left(g_{0}, \ldots, g_{k-1}\right)$ and a query $q=\langle S, f n m\rangle$, the answer $\alpha_{F Q L}(\bar{g}, q)$ is defined iff

1. fnm denotes a built-in function, or

2. $f n m$ denotes one of the stored functions, i.e. $f n m=n a m e\left(g_{i}\right)$, or

3. $f n m$ denotes a derived function specified by $S$ and $S$ satisfies the three conditions of Proposition 9.

In the first case, the answer $\alpha_{F Q L}(\bar{g}, q)$ is equal to the built-in function named $f n m$. This answer is independent of the stored functions and from the derived functions. In the second case, $\alpha_{F Q L}(\bar{g}, q)=g_{i}$ if $f n m=\operatorname{name}\left(g_{i}\right)$. In this case, the answer is independent of the derived functions. In the third case, $\alpha_{F Q L}(\bar{g}, q)=f_{i}$ if $f n m=\operatorname{name}\left(f_{i}\right)$ and the derived function $f_{i}$ is determined by the unique minimum solution of the system of inequalities corresponding to $S$. The derived functions may depend on the stored functions as well as on the built-in functions.

The correspondences between the instances of the databases and the correspondences between the queries are defined as follows. Let

$$
I S_{F O+L F P}=\left\langle D B S_{r e l}, Q L_{F O+L F P}, A S_{r e l}, \alpha F O+L F P\right\rangle
$$


be the relational database system which is accessible through fixpoint queries as described in Section 3. The function

$$
u: D B S_{\text {rel }} \rightarrow D B S_{F Q L},^{\prime}(D, \bar{R}) \mapsto \bar{g}
$$

assigns every instance $(D, \bar{R})$ a tuple of stored functions $u(D, \bar{R})=$ $\left(g_{0}, \ldots, g_{k-1}\right)$ such that $R_{i} \simeq g_{i}$ for $0 \leq i<k$ where as usual, $\bar{R}=$ $\left(R_{0}, \ldots, R_{k-1}\right)$. The function

$$
v: \operatorname{range}\left(\alpha_{F O+L F P}\right) \rightarrow \operatorname{range}\left(\alpha_{F Q L}\right), R \mapsto f
$$

assigns every answer $R \subseteq D^{b}$ a function $f: D^{b} \rightarrow B(\{1\})$ such that $R \simeq f$. With respect to the correspondences given by $u$ and $v$, we have shown that FQL* is more expressive than FO+LFP.

Proposition 11 The query language $Q L_{F Q L}$ of $I S_{F Q L}$ is more expressive than $Q L_{F O+L F P}$ of $I S_{F O+L F P}$ with respect to the correspondences given by $u$ and $v$, i.e.

$$
Q L_{F O+L F P}\left(I S_{F O+L F P}\right)<_{u, v} \quad Q L_{F Q L}\left(I S_{F Q L}\right)
$$

Proof. At the outset of this section, it was shown that for every fixpoint query $q \in Q L_{F O+L F P}$, there exists a query $h(q) \in Q L_{F Q L}$ such that $\alpha_{F O+L F P}((D, \bar{R}), q) \simeq \alpha_{F Q L}(u(D, \bar{R}), h(q))$. This implies that $Q L_{F O+L F P}\left(I S_{F O+L F P}\right) \leq_{u, v} Q L_{F Q L}\left(I S_{F Q L}\right)$. Finally, from $Q L_{F O+L F P}\left(I S_{F O+L F P}\right)={ }_{u, v} Q L_{F Q L}\left(I S_{F Q L}\right)$ would follow that the fixpoint queries were able to count. Since, this is not the case, $Q L_{F Q L}$ is more expressive than $Q L_{F O+L F P} . \diamond$

\section{Conclusions}

It was argued that a new notion of expressive power is needed to compare non-relational query languages, particularly, advanced query languages with counting abilities. The proposed new notion of expressive power has been shown to facilitate the comparison of the expressive power of such query languages. When restricted to relational query languages, the new notion of expressive power is equivalent to the notion of expressive power by Chandra and Harel.

The new notion of expressive power has been serving as a platform to derive new results. First, the expressive power of a Boolean query language has been compared with the expressive power of a weighted 
query language. Second, FQL* has been presented as an example of a non-relational query language with an outstanding expressive power. We described only those parts of the query language FQL* that are concerned with its expressive power. It was shown that the expressive power of FQL* surpasses the expressive power of fixpoint queries. This result is mainly due to the use of bag functions which encompass a primitive form of counting together with a primitive form of infinity. Thus, the complete lattice of bag functions may serve as an interesting alternative to the conventional power set of Herbrand interpretations.

\section{A Theorems and Proofs}

Proposition 6 Let $q_{b}$ be any Boolean query and let $J_{q}$ be the set of indices of those items $d_{j}$ that satisfy $q_{b}$, i.e. $J_{q}:=\left\{j \mid d_{j} \in \alpha_{b}\left(A, q_{b}\right)\right\}$. There exists a weighted query $q_{w}$ such that for all data items $d_{j}$ in $D$,

$$
R S V_{w}\left(q_{w}, d_{j}\right)= \begin{cases}1.0 & \text { if } d_{j} \in \alpha_{b}\left(A, q_{b}\right) \\ 0.0 & \text { otherwise }\end{cases}
$$

if and only if

$$
\begin{gathered}
j \in J_{q} \wedge d_{j}=\sum_{h \in J_{q}-\{j\}} c_{h} d_{h} \Longrightarrow \sum_{h \in J_{q}-\{j\}} c_{h}=1, \\
j \notin J_{q} \wedge d_{j}=\sum_{h=0}^{n-1} c_{h} d_{h} \Longrightarrow \sum_{h \in J_{q}} c_{h}=0 .
\end{gathered}
$$

Praof. We define a Boolean retrieval function $R S V_{b}: Q L_{b} \times D \rightarrow \mathrm{R}$ as follows.

$$
R S V_{b}\left(q_{b}, d_{j}\right):= \begin{cases}1.0 & \text { if } d_{j} \in \alpha_{b}\left(A, q_{b}\right) \\ 0.0 & \text { otherwise }\end{cases}
$$

Then, condition (74) is equivalent to $R S V_{b}\left(q_{b}, d_{j}\right)=R S V_{w}\left(q_{w}, d_{j}\right)$ for all data items $d_{j}$ in $D$.

First, we show how (75) and (76) imply the existence of a weighted query $q_{w}$ such that for all $d_{j} \in D, R S V_{b}\left(q_{b}, d_{j}\right)=R S V_{w}\left(q_{w}, d_{j}\right)$. According to (26), every item $d_{j}$ is regarded as a column vector of the feature-item matrix $A$. Let $q_{b}$ be any Boolean query. In what follows, we show the existence of a corresponding query $q_{w}$. Without loss of generality, we assume that the items are numbered in such a way that 
- the vectors $d_{0}, \ldots, d_{u-1}, d_{u}, \ldots, d_{u+v-1}$ are linearly independent,

- the items $d_{0}, \ldots, d_{u-1}$ satisfy the query $q_{b}$,

- the items $d_{u}, \ldots, d_{u+v-1}$ do not satisfy the query $q_{b}$,

- every vector $d_{j}$ that satisfies the query $q_{b}$ is a linear combination of the vectors $d_{0}, \ldots, d_{u-1}$, and

- every vector $d_{j} \in D$ is a linear combination of $d_{0}, \ldots, d_{u+v-1}$.

. It is easy to show that the items can always be numbered in such a way.

Let $A_{u+v}$ be the submatrix of $A$ consisting of the first $u+v$ columns. Since the columns of $A_{u+v}$ are linearly independent and $M$ is positive definite, the columns of $M A_{u+v}$ are also linearly independent. Thus, $M A_{u+v}$ contains $u+v$ linearly independent rows.

Without loss of generality, we assume that the first $u+v$ rows of $M A_{u+v}$ are linearly independent. The submatrix of $M A_{u+v}$ consisting of the first $u+v$ rows is denoted by $B$. The matrix $B$ is a regular $(u+v) \times(u+v)$ matrix which is also a submatrix of $M A$.

$$
M A=\begin{array}{|l|l|}
\hline B \\
\hline
\end{array}
$$

Since $B$ is regular, the inverse matrix $B^{-1}$ exists. The matrix $C$ is obtained by adding $m-u-v$ zero vectors to the right of $B^{-1}$.

$$
\begin{array}{|l|l|}
C:=B^{-1} & 0 \\
\hline
\end{array}
$$

The $(u+v)$-dimensional row vector $r^{T}$ consists of $u$ ones and of $v$ zeros.

$$
r^{T}:=(1, \ldots, 1,0, \ldots, 0)
$$

When multiplying $C$ and $M A$, we obtain a $(u+v) \times n$ matrix where the left square corresponds to the unit matrix $E$.

$$
C M A=\begin{array}{|l|l|}
\hline E & * \\
\hline
\end{array}
$$


When multiplying $r^{T}$ and $C M A$, we obtain an $n$-dimensional row vector where the first $u$ components are equal to 1 and the next $v$ components are equal to zero.

$$
r^{T} C M A=(1, \ldots, 1,0, \ldots, 0, *, \ldots, *)
$$

We define $q_{w}:=C^{T} r$. To show that for all $d_{j} \in D, R S V_{b}\left(q_{b}, d_{j}\right)=$ $R S V_{w}\left(q_{w}, d_{j}\right)$, we distinguish four cases. Let $d_{j} \in D$ be any item.

Case I: $j \in\{0, \ldots, u-1\}$. Since $d_{j}$ is the $j^{t h}$ column of $A$, $R S V_{w}\left(q_{w}, d_{j}\right)=q_{w}^{T} M d_{j}=r^{T} C M d_{j}$ is equal to the $j^{t / 2}$ component of $r^{T} C M A$ which is equal to $1=R S V_{b}\left(q_{b}, d_{j}\right)$.

Case II: $j \in\{u, \ldots, u+v-1\}$. Similarly to case I, $R S V_{w}\left(q_{w}, d_{j}\right)$ is equal to the $j^{t h}$ component of $r^{T} C M A$ which is equal to $0=$ $R S V_{b}\left(q_{b}, d_{j}\right)$.

Case III: $j \in\{u+v, \ldots, n-1\}$ and $d_{j}$ satisfies $q_{b}$, i.e. $R S V_{b}\left(q_{b}, d_{j}\right)=$ 1. Since $d_{j}$ is not a base vector, $d_{j}=\sum_{h=0}^{u-1} c_{h} d_{h}$. From the linearity of $R S V_{w}$ and from (75) follows $R S V_{w}\left(q_{w}, d_{j}\right)=\sum_{h=0}^{u-1} c_{h} R S V_{w}\left(q_{w}, d_{h}\right)=$ $\sum_{h=0}^{u-1} c_{h}=1=R S V_{b}\left(q_{b}, d_{j}\right)$.

Case IV: $j \in\{u+v, \ldots, n-1\}$ and $d_{j}$ does not satisfy $q_{b}$, i.e. $R S V_{b}\left(q_{b}, d_{j}\right)=0$. Since $d_{j}$ is not a base vector and $d_{j}=\sum_{h=0}^{u+v-1} c_{h} d_{h}$. From the linearity of $R S V_{w}$ and finally, from (76) follow the equalities $R S V_{w}\left(q_{w}, d_{j}\right)=\sum_{h=0}^{u+v-1} c_{h} R S V_{w}\left(q_{w}, d_{h}\right)=\sum_{h=0}^{u-1} c_{h} 1+\sum_{h=u}^{u+v-1} c_{h} 0$ $=0=R S V_{b}\left(q_{b}, d_{j}\right)$.

Second, we show that if (75) and (76) are not satisfied, the existence of a weighted query $q_{w}$ such that $R S V_{w}\left(q_{w}, d_{j}\right)=R S V_{b}\left(q_{b}, d_{j}\right)$ yields a contradiction.

Assume that (75) is not satisfied, i.e. there exists a $j$ such that $j \in$ $J_{q}, d_{j}=\sum_{h \in J_{q}-\{j\}} c_{h} d_{h}$, and $\sum_{h \in J_{q}-\{j\}} c_{h} \neq 1$. From the linearity of $R S V_{w}$ we get a contradiction: $1=R S V_{b}\left(q_{b}, d_{j}\right)=R S V_{w}\left(q_{w}, d_{j}\right)=$ $\sum_{h \in J_{q}-\{j\}} c_{h} R S V_{w}\left(q_{w}, d_{h}\right)=\sum_{h \in J_{q}-\{j\}} c_{h} \neq 1$.

Assume that (76) is not satisfied, i.e. there exists a $j$ such that $j \notin J_{q}, d_{j}=\sum_{h=0}^{n-1} c_{h} d_{h}$, and $\sum_{h \in J_{q}} c_{h} \neq 0$. Again, from the linearity of $R S V_{w}$ there follows a contradiction: $0=R S V_{b}\left(q_{b}, d_{j}\right)=$ $R S V_{w}\left(q_{w}, d_{j}\right)=\sum_{h=0}^{n-1} c_{h} R S V_{w}\left(q_{w}, d_{h}\right)=\sum_{h \in J_{q}} c_{h} * 1+\sum_{h \notin J_{q}} c_{h} * 0=$ $\sum_{h \in J_{q}} c_{h} \neq 0$. $\diamond$

Proposition 9 Assume that the functions $f_{0}, \ldots, f_{n-1}$ are specified by $n$ create statements which correspond to a system of $n$ inequalities of the form

$$
f_{i}\left(\bar{x}_{i}\right) \sqsupseteq \chi_{i}\left(\pi_{i}\left(\sigma\left(\varphi_{i}(\bar{f}), \bar{x}_{i}\right)\left(X(i, 0) \times \ldots \times X\left(i, s_{i}-1\right)\right)\right)\right) .
$$


This system of $n$ inequalities has a uniquely determined minimum solution if there exists a stratification function

$$
\nu:\{0, \ldots, n-1\} \rightarrow\{0, \ldots, n-1\}, i \mapsto \nu(i)
$$

such that for every $i \in\{0, \ldots, n-1\}$, the following three conditions are satisfied.

1. If $f_{j}$ occurs in the specification of $f_{i}$, then $\nu(j) \leq \nu(i)$.

2. The domain $\left.X(i, 0) \times \ldots \times X\left(i, q_{i}-1\right)\right)$ of $f_{i}$ is finite.

3. If $f_{j}$ occurs in the specification of $f_{i}$ and $\nu(j)=\nu(i)$, then $\varphi_{i}$ is monotonic and $Y(i, 0) \times \ldots \times Y\left(i, p_{i}-1\right)$ is finite.

The uniquely determined minimum solution is computable by a polynomial time algorithm, i.e. in time $O(P(|X|))$ where $P$ denotes a polynomial and $|X|$ denotes the number of objects stored in the database.

Proof. The proof of this proposition is not particularly complicated even though it is several pages long. The semantics of FQL* is based on a non-Boolean distributive lattice whereas the semantics of conventional query languages is based on Boolean lattices, e.g. the set of Herbrand interpretations. Thus, we have to develop a new theoretical framework from scratch. For this reason the following proof is quite long.

We first introduce some notations which will be used subsequently. We will say that a function $f_{i}$ or an inequality $f_{i}(\ldots) \sqsupseteq \chi_{i}(\ldots)$ belongs to the stratification level $k$ iff $\nu(i)=k$. In what follows, the definitions of least solutions and minimum solutions are given. Let $L$ be the set of $n$-tuples $\bar{f}=\left(f_{0}, \ldots, f_{n-1}\right)$ where $f_{i}$ is a function mapping $X(i, 0) \times \ldots \times X\left(i, q_{i}-1\right)$ to $B\left(Y(i, 0) \times \ldots \times Y\left(i, p_{i}-1\right)\right)$. Using (53), we define $\bar{f} \sqsubseteq \bar{f}^{\prime}$ iff $f_{i} \sqsubseteq f_{i}^{\prime}$ for $0 \leq i<n$. The structure $\langle L, \sqsubseteq\rangle$ is a partial ordering because $\sqsubseteq$ is reflexive, antisymmetric, and transitive. The $n$-tuple $\bar{f}$ is called a solution iff $\bar{f}$ satisfies the specified $n$ inequalities. The $n$-tuple $\bar{f}$ is called a least solution iff it is a solution and every solution $\bar{f}^{\prime}$. entails $\bar{f}$, i.e. $\bar{f}^{\prime} \sqsupseteq \bar{f}$. Let $\bar{f}$ and $\bar{f}^{\prime}$ be least solutions. By definition, $\bar{f} \sqsubseteq \bar{f}^{\prime}$ and $\bar{f}^{\prime} \sqsubseteq \bar{f}$ imply $\bar{f}=\bar{f}^{\prime}$ because $\sqsubseteq$ is antisymmetric. Hence, if there exists a least solution then it is unique. The $n$-tuple $\bar{f}$ is called a minimum solution iff there exists no solution $\bar{f}^{\prime}$ which is less than $\bar{f}$, i.e. $\bar{f}^{\prime} \not \subset \bar{f}$ for every solution $\bar{f}^{\prime}$. Every least solution is a minimum solution but not vice versa. 
Next, we show that the inequalities of every stratification level $k$ have a least solution if the functions of the lower stratification levels $0, \ldots, k-1$ are already computed. Note that the least solution of the inequalities of a stratification level determines the derived functions of this stratification level. Hence, computing the least solution of the inequalities of a stratification level is equivalent to computing the derived functions of this stratification level. The derived functions of the lower levels can be considered as stored functions. We assume without restricting the generality that there is only one stratification level containing $n$ inequalities. In what follows, we show the existence of a least solution of these inequalities.

Assume that there exists an index $i \in\{0, \ldots, n-1\}$ such that $\varphi_{i}$ is non-monotonic or $Y(i, 0) \times \ldots \times Y\left(i, p_{i}-1\right)$ is infinite. From the third condition of the proposition follows that the specification of the function $f_{i}$ does not depend on the functions $f_{0}, \ldots, f_{n-1}$. In particular, $\varphi_{i}(\bar{f})$ does not depend on $\bar{f}$ nor does $\chi_{i}$ depend on $\bar{f}$. Hence, the functions $\chi_{i}$ and $\pi_{i}$ and the predicates $\varphi_{i}\left(\bar{f}, \bar{x}_{i}\right)$ are well defined. In this case, the least solution of the inequality $f_{i}(\ldots) \sqsupseteq$ $\chi_{i}(\ldots)$ is obviously given by

$$
f_{i}\left(\bar{x}_{i}\right):=\lambda_{i}\left(\pi_{i}\left(\sigma\left(\varphi_{i}(\bar{f}), \bar{x}_{i}\right)\left(X(i, 0) \times \ldots \times X\left(i, s_{i}-1\right)\right)\right)\right.
$$

and the function $f_{i}$ is considered as a stored function like the derived functions of the lower stratification levels. We proceed by restarting with a smaller set of functions, i.e. $\left\{f_{0}, \ldots, f_{i-1}, f_{i+1}, \ldots, f_{n-1}\right\}$. If there exists a $j \in\{0, \ldots, i-1, i+1, \ldots, n-1\}$ such that $\varphi_{j}$ is non-monotonic, $\chi_{j}$ contains a non-monotonic non-derived function, or $Y(j, 0) \times \ldots \times Y\left(j, p_{j}-1\right)$ is infinite, then $f_{j}$ is also considered as a stored function. This procedure is repeated until the set of derived functions is empty or for each remaining $f_{k}, \varphi_{k}$ is monotonic and $Y(k, 0) \times \ldots \times Y\left(k, p_{k}-1\right)$ is finite.

Hence, we may assume that for every $f_{i} \in\left\{f_{0}, \ldots, f_{n-1}\right\}, \varphi_{i}$ is monotonic and $Y(i, 0) \times \ldots \times Y\left(i, p_{i}-1\right)$ is finite. We define a function

$$
T: L \rightarrow L, \bar{f} \mapsto\left(T_{0}(\bar{f}), \ldots, T_{n-1}(\bar{f})\right)
$$

where $T_{i}(\bar{f})$ and $f_{i}$ are functions of the same type.

$$
\begin{aligned}
T_{i}(\bar{f}): X(i, 0) \times \ldots X\left(i, q_{i}-1\right) & \rightarrow B\left(Y(i, 0) \times \ldots Y\left(i, p_{i}-1\right)\right), \\
\bar{x}_{i} & \mapsto T_{i}(\bar{f})\left(\bar{x}_{i}\right)
\end{aligned}
$$

The function $T_{i}(\bar{f})$ is defined by

$$
T_{i}(\bar{f})\left(\bar{x}_{i}\right):=\chi_{i}\left(\pi _ { i } \left(\sigma\left(\varphi_{i}\left(\bar{f}, \bar{x}_{i}\right)\left(X(i, 0) \times \ldots \times X\left(i, s_{i}-1\right)\right)\right) .\right.\right.
$$


Since $\varphi_{i}$ is monotonic, $\bar{f} \sqsubseteq \bar{f}^{\prime}$ implies that

$$
\begin{aligned}
& \sigma\left(\varphi_{i}(\bar{f}), \bar{x}_{i}\right)\left(X(i, 0) \times \ldots X\left(i, s_{i}-1\right)\right) \\
& \quad \sqsubseteq \sigma\left(\varphi_{i}\left(\bar{f}^{\prime}\right), \bar{x}_{i}\right)\left(X(i, 0) \times \ldots X\left(i, s_{i}-1\right)\right) .
\end{aligned}
$$

Furthermore, $\pi_{i}$ and $\chi_{i}$ are always monotonic. Thus, $\bar{f} \sqsubseteq \bar{f}^{\prime}$ implies that $T_{i}(\bar{f}) \sqsubseteq T_{i}\left(\bar{f}^{\prime}\right)$ and hence, $T$ is monotonic.

Let $\langle L, \subseteq\rangle$ be the partial ordering introduced above. Given any subset $M \subseteq L$, we define $\bar{g}=\left(g_{0}, \ldots, g_{n-1}\right) \in L$ such that

$$
g_{i}\left(\bar{x}_{i}\right):=\bigsqcup_{\left(f_{0} \ldots f_{n-1}\right) \in M} f_{i}\left(\bar{x}_{i}\right) .
$$

It is easy to show that $\bar{g}$ is the least upper bound of $M$, i.e. $\bar{g}=\operatorname{lub}(M)$. The existence of the greatest lower bound $g l b(M)$ for every $M \subseteq L$ is shown similarly. Thus, $\langle L, \sqsubseteq\rangle$ is a complete lattice and according to a theorem by Tarski [25], the monotonic function $T: L \rightarrow L$ has a least fixpoint called $l f p(T)$. Obviously, this least fixpoint is the least solution of the $n$ inequalities. Remember that, for simplicity, we assumed that all inequalities belong to the same stratification level. This completes the proof for the existence of a least solution for every stratification level.

Subsequently, we consider the general case, where the derived functions $f_{0}, \ldots, f_{n-1}$ belong to different stratification level. We will show that the bottom-up computation that starts at the stratification level 0 and ends at the stratification level $n-1$ provides a unique minimum solution of the $n$ inequalities. This bottom-up procedure provides a solution because all inequalities are satisfied. The solution thus obtained is a minimum solution because, otherwise, the subsolutions of the particular stratification levels were not the least solutions. The order in which the derived functions are computed depends on the stratification function. Thus, the minimum solution seems to depend on the particular stratification function. This is not the case and it can be proven in the same way as it is proven that the minimum Herbrañ model of a stratified Datalog program does not depend on the stratification function $[3$, Theorem 11].

In what follows, we present an algorithm to determine the minimum solution. Again, we consider only one stratification level and we assume that all derived functions $f_{0}, \ldots, f_{n-1}$ belong to this level. Furthermore, to keep the notation simple, we assume that there is only 
one class of objects called $X$ and every derived function is of the form $f_{i}: X \rightarrow B(X)$.

An iteration procedure is used to compute the least solution. Every function $f_{i}$ is assigned a table $\tau_{i}$. At the outset, every table $\tau_{i}$ contains for every $x, y \in X$ an entry $(x, y, 0,0)$. The tables are updated when the iteration step is in progress. For every iteration step $s$, the tables $\tau_{0}, \ldots, \tau_{n-1}$ determine an element $\bar{f}^{(s)}=\left(f_{0}^{(s)}, \ldots, f_{n-1}^{(s)}\right) \in L$ by

$$
f_{i}^{(s)}(x)(y)=m \quad \Longleftrightarrow \quad(x, y, m, k) \in \tau_{i} .
$$

Given an entry $(x, y, m, k) \in \tau_{i}$, the number $k$ refers to the last iteration step when this entry has been updated.

Assume that $s$ iteration steps are already performed. The next iteration step, $s+1$, is performed as follows. We distinguish two cases. First, if $s$ divides $n|X|^{2}$, we compute the graph $\langle V, E\rangle$ defined by $V:=\{0, \ldots, n-1\} \times X \times X$ and

$$
\begin{aligned}
& ((i, v, w),(j, x, y)) \in E \\
& \quad: \Longleftrightarrow T_{j}\left(\mu(i, v, w)\left(\bar{f}^{(s)}\right)\right)(x)(y)>T_{j}\left(\bar{f}^{(s)}\right)(x)(y) .
\end{aligned}
$$

The function $\mu(i, v, w): L \rightarrow L, \bar{f} \mapsto \mu(i, v, w)(\bar{f})$ is defined by

$$
\begin{aligned}
\mu(i, v, w) & :=\left(\mu_{0}(i, v, w), \ldots, \mu_{n-1}(i, v, w)\right) \\
\mu_{h}(i, v, w)(\bar{f})(t)(u) & := \begin{cases}f_{i}(v)(w)+1 & \text { if }(i, v, w)=(h, t, u) \\
f_{h}(t)(u) & \text { otherwise }\end{cases}
\end{aligned}
$$

Note that $\mu(i, v, w)(\bar{f})$ is identical to $\bar{f}$ except that the bag $f_{i}(v)$ contains an additional element $w$. An edge from $(i, v, w)$ to $(j, x, y)$ represents the following dependency between $f_{i}$ and $f_{j}$. If a future iteration step requires an increment of $f_{i}(v)(w)$ then $f_{j}(x)(y)$ has to be incremented as well. In other words, if in one of the subsequent iteration steps, $w$ is added to $f_{i}(v)$, then at least one element $y$ has to be added to $f_{j}(x)$.

Let us assume that $s$ divides $n|X|^{2}$ and the graph $\langle V, E\rangle$ has been computed. Then, every entry $(x, y, m, k)$ in the table $\tau_{j}$ is replaced by the entry $(x, y, \omega, s+1)$ if the vertex $(j, x, y)$ belongs to a cycle and $T_{j}\left(\bar{f}^{(s)}\right)(x)(y)>T_{j}\left(\bar{f}^{(s-1)}\right)(x)(y)$. In this case, $\bar{f}^{(s-1)} \sqsubseteq l f p(T)$ implies that $f_{j}(x)(y)=\omega$ is a necessary condition for $\bar{f}=\operatorname{lf} p(T)$. Hence, from $\bar{f}^{(s)} \sqsubseteq \operatorname{lf} p(T)$ follows that $\bar{f}^{(s+1)} \sqsubseteq l f p(T)$. 
In the second case, where $s$ does not divide $n|X|^{2}$, an entry $(x, y, m, k)$ in $\tau_{j}$ is replaced by the entry $\left(x, y, m^{\prime}, s+1\right)$ whenever $m^{\prime}=T_{j}\left(\bar{f}^{(s)}\right)(x)(y)>m$. Because $T$ is monotonic, from $\bar{f}^{(s)} \sqsubseteq \operatorname{lf} p(T)$ follows again that $\bar{f}^{(s+1)} \sqsubseteq l f p(T)$.

We have considered two different types of iteration steps depending on whether $s$ divides $n|X|^{2}$ or not. In both cases,

$$
\bar{f}^{(s)} \sqsubseteq l f p(T) \Longrightarrow \bar{f}^{(s+1)} \sqsubseteq l f p(T) .
$$

Since $\bar{f}^{(0)} \sqsubseteq l f p(T)$, it follows that $\bar{f}^{(s)} \sqsubseteq l f p(T)$ for every iteration step $s$.

When no new entries are inserted, the iteration is terminated. If $t$ is the last iteration step, $\bar{f}^{(t)}$ is a fixpoint. This implies $l f p(T) \sqsubseteq \bar{f}^{(t)}$. Together with $\bar{f}^{(t)} \sqsubseteq l f p(T)$ it follows that the iteration procedure provides the least fixpoint because $\bar{f}^{(t)}=l f p(T)$.

It remains to show that the iteration procedure is a polynomial time algorithm. Let us consider paths without cycles in the graph $G$. The longest path without cycles along which an increment can be propagated is $|V|-1$. Thus, if the iteration procedure is not terminated after $|V|=n|X|^{2}$ iteration steps, the graph $G$ contains cycles. In this case, at least one entry $(x, y, \omega, s)$ is inserted into a table and this entry is no more changed in the subsequent iteration steps. Since the number of entries is equal to $|V|$, we have an upper bound $|V|^{2}=n^{2}|X|^{4}$. Hence, the iteration procedure is a polynomial time algorithm. $\diamond$

\section{References}

[1] A. V. Aho and J. D. Ullman. Universality of Data Retrieval Langages. In Symposium on Principles of Programming Languages, pages 110-117, 1979.

[2] J. Albert. Algebraic Properties of Bag Data Types. In International Conference on Very Large Data Bases, 1991.

[3] K. R. Apt, H. A. Blair, and A. Walker. Towards à Theory of Declarative Knowledge. In J. Minker, editor, Foundations of Deductive Databases and Logic Programming, Los Altos, California, 1988. Morgan Kaufmann Publishers. 
[4] F. Baader. A Formal Definition for the Expressive Power of Knowledge Representation Languages. In L. C. Aiello, editor, European Conference on Artificial Intelligence, pages 53-58, London, 1990. Pitman.

[5] D. Barbara, H. Garcia-Molina, and D. Porter. A Probabilistic Relational Model. In Advancès in Database Technology-EDBT'90, pages 60-74, Berlin, 1990. Springer-Verlag.

[6] D. Beech. A Foundation for Evolution from Relational to Object Databases. In Advances in Database Technology-EDBT'88, pages 251-270, Berlin, 1988. Springer-Verlag.

[7] G. Birkhoff and J. D. Lipson. Heterogeneous Algebras. Journal of Combinatorial Theory, 8:115-133, 1970.

[8] D.A. Buell and D.H. Kraft. A Model for a Weighted Retrieval System. Journal of the ASIS, 32(3):211-216, 1981.

[9] A. Chandra and D. Harel. Computable Queries for Relational Databases. Journal of Computer and System Sciences, 21(2):156178, 1980.

[10] A. Chandra and D. Harel. Structure and Complexity of Relational Queries. Journal of Computer and System Sciences, 25(1):99-128, 1982.

[11] A. K. Chandra. Theory of Database Queries. In Symposium on Principles of Database Systems (PODS), pages 1-9, 1988.

[12] E. F. Codd. Relational Completeness of Database Sublanguages. In Rustin; editor, Database Systems. Prentice-Hall, 1972.

[13] M. Davis. Unsolvable Problems. In J. Barwise, editor, Handbook of Mathematical Logic. North-Holland, 1977.

[14] L.G. DeMichiel. Resolving Database Incompatibility: An Approach to Performing Relational Operations over Mismatched Domains. IEEE Transactions on Knowledge and.Data Engineering, 1(4):485-493, 1989.

[15] N. Fuhr. A Probabilistic Framework for Vague Queries and Imprecise Information in Databases. In D. McLeod, R. Sacks-Davis, and H. Schek, editors, International Conference on Very Large Data Bases, pages 696-707, Los Altos, CA, 1990. Morgan Kaufman. 
[16] M. R. Garey and D. S. Johnson. Computers and Intractability, A Guide to the Theory of NP-Completeness. W. H. Freeman and Company, New York, NY, 1979.

[17] Y. Gurevich. Toward Logic Tailored for Computational Complexity. In Computation and Proof Theory, Lecture Notes in Mathematics, pages 175-216. Springer-Verlag, 1984.

[18] Y. Gurevich and S. Shela. Fixpoint Extensions of First-Order Logic. Annals of Pure and Applied Logic, 32:265-280, 1986.

[19] M. A. W. Houtsma, P. M. G. Apers, and S. Ceri. Complex Transitive Closure Queries on a Fragmental Graph. In International Conference on Database Theory, pages 470-484. Springer-Verlag, 1990.

[20] N. Immerman. Relational Queries Computable in Polynomial Time. Information and Control, 68:86-104, 1986.

[21] P. Kolaitis. The Expressive Power of Stratified Logic Programs. Information and Control, 90(1):50-66, 1991.

[22] G. Salton and M.J. McGill. Introduction to Modern Information Retrieval. McGraw-Hill, New York, 1983.

[23] L. V. Saxton and V. V. Raghavan. Design of an Integrated Information Retrieval/Database Management System. IEEE Transactions on Knowledge and Data Engineering, 2(2):210-219, 1990.

[24] P. Schäuble. On the Compatibility of Retrieval Functions, Preference Relations, and Document Descriptions. Technical Report 113, ETH Zurich, Department of Computer Science, 1989.

[25] A. Tarski. A Lattice-Theoretical Fixpoint Theorem and Its Applications. Pacific Journal of Mathematics, 5:285-309, 1955.

[26] J.D. Ullman. Principles of Database Systems. Computer Science Press, Rockville, Maryland, second edition, 1982.

[27] C. J. van Rijsbergen. Information Retrieval. Butterworths, London, second edition, 1979.

[28] K. Wilkinson, P. Lyngbæk, and W. Hasan. The Iris Architecture and Implementation. IEEE Transactions on Knowledge and Data Engineering, 2(1):63-75, 1990. 
[29] B. Wüthrich. Large Deductive Databases with Constraints. $\mathrm{PhD}$ thesis, Swiss Federal Institute of Technology, 1991. VdF-Verlag, Zürich. 
Gelbe Berichte des Departements Informatik

155 D. Crippa

156 R. Griesemer

C. Pfister (ed.), B. Heeb,

J. Templ

157 T. Weibel, G. Gonnet

158 M. Scholl (ed.)

159 K. Gates

160 H. Mössenböck

161 S. Lalis

162 G. Weikum, C. Hasse

163 J. Nievergelt et al.

164 G.H. Gonnet, H. Straub

165 L. Adams, P. Arbenz

166 E. Margulis

167 H.E. Meier

168 B. Heeb, I. Noack

169 M. Bronstein

170 P. Arbenz

171 K. Simon, P. Trunz

172 A. Rosenthal, Ch. Rich, M.H. Scholl
A Special Case of the Dynamization Problem for Least Cost Paths

On the Linearization of Graphs and Writing Symbol

Files

Oberon Technical Notes

An Algebra of Properties

Grundlagen von Datenbanken (Kurfassungen

des 3.GI-Workshops, Volkse, 21. - 24.5.91)

Using Inverse Iteration to Improve the Divide and Conquer Algorithm

Differences between Oberon and Oberon-2

The programming Language Oberon-2 (vergriffen)

XNet: Supporting Distributed Programming in the Oberon Environment (vergriffen)

Multi-Level Transaction Management for Complex Objects: Implementation, Performance, Parallelism

eXperimental geometrY Zurich: Software for Geometric Computation

The Dynamic Programming Algorithm as a Finite Automation

Towards a Divide and Conquer Algorithm for the Real Nonsymmetric.Eigenvalue Problem

N-Poisson Document Modelling Revisited

Schriftgestaltung mit Hilfe des Computers

Typographische Grundregeln mit Gestaltungsbeispielen (neue erweiterte Auflage)

Hardware Description of the Workstation Ceres-3

Formulas for Series Computations

Divide and Conquer Algorithms for the Bandsymmetric Eigenvalue Problem

On Transitive Orientation

Reducing Duplicate Work in Relational Join(s): A Unified Approach 